\title{
Renormalization of composite operators
}

\author{
J. Polonyi \\ Institute for Theoretical Physics, Louis Pasteur University, Strasbourg, France \\ and Department of Atomic Physics, Lorand Eötvös University, Budapest, Hungary \\ K. Sailer \\ Department for Theoretical Physics, University of Debrecen, Debrecen, Hungary
}

(Received 10 November 2000; published 19 April 2001)

\begin{abstract}
The blocked composite operators are defined in the one-component Euclidean scalar field theory, and shown to generate a linear transformation of the operators, the operator mixing. This transformation allows us to introduce the parallel transport of the operators along the renormalization group trajectory. The connection on this one-dimensional manifold governs the scale evolution of the operator mixing. It is shown that the solution of the eigenvalue problem of the connection gives the various scaling regimes and the relevant operators there. The relation to perturbative renormalization is also discussed in the framework of the $\phi^{3}$ theory in dimensions $d=6$.
\end{abstract}

DOI: 10.1103/PhysRevD.63.105006

PACS number(s): 11.15.Tk, 11.10.Gh

\section{INTRODUCTION}

The renormalization group $(\mathrm{RG})$ strategy is to follow the evolution of the coupling constants in the observational scale in order to identify the important interactions at different scales. This is achieved by blocking, the successive lowering of the UV cutoff, and tracing the resulting blocked, renormalized action [1,2]. There is an alternative method, implicit in this procedure, where the original cutoff and action are kept but the operators are modified to cover less modes while keeping the expectation values fixed. This paper outlines this latter method in a more systematic manner and compares it with the traditional one.

A similar question arises in the renormalization of composite operators where the goal is to render the Green's functions containing the insertion of some local operators that are not in the action finite as the cutoff is removed. The perturbative treatment of this problem proceeds by the introduction of additional counterterms in the action in such a manner that the original Green's functions remain unchanged but those with the insertion of the composite operators turn out to be finite [3-6].

This rather complicated procedure attempts to perform an amazing project: the renormalization of an otherwise nonrenormalizable model. This happens because the insertion of the nonrenormalizable composite operators in the Green's functions can be achieved by introducing them in the action with a source term and taking the derivative of the logarithm of the partition function with respect to their source. As long as the Green's functions are finite so is the partition function containing the nonrenormalizable operators. The resolution of this apparent paradox is that the rendering of more Green's functions with the composite operator insertion finite requires more counterterms. At the end we should need infinitely many counterterms to complete the project, just as when we would attempt to remove the cutoff in a nonrenormalizable model.

In the traditional multiplicative renormalization of quantum field theory the irrelevant, nonrenormalizable coupling constants are ignored. In fact, the derivation of the renormalization group equations in renormalizable-quantum-field theory is based on the compensation of the change of the scale by the adjusting of the relevant (or marginal) coupling constants. This procedure is made insensitive to the irrelevant terms by considering the asymptotic scaling regime only and by ignoring the vanishing contributions as the cutoff is removed. It cannot help us to understand crossovers or the competition of different fixed points, in general. One might object that irrelevant operators do appear in the renormalization of composite operators within the traditional multiplicative renormalization scheme. But their evolution is determined by the requirement that the Feynman graphs considered remain finite. This prescription misses the finite, physical part of the renormalization. The blocking procedure, followed in the present paper provides a renormalization scheme where the evolution of all coupling constants is well defined and we are not confined into the asymptotic scaling regimes.

The nonperturbative renormalization due to blocking has proven to be effective in establishing renormalizability as well. The old-fashioned perturbative proof of renormalizability that is made involved by the tracing of overlapping divergences [6] is simplified enormously by considering a nonperturbative renormalization scheme $[7,8]$. In fact, the renormalizability turns out to be the absence of the UV Landau pole. This pole can easily be avoided in the framework of the loop expansion for asymptotically free models.

Our technique to follow the mixing of composite operators by adding them to the action has already been used in the framework of the perturbative, multiplicative renormalization group scheme. In the perturbative studies of the renormalizability of Green's functions one or more composite operators were inserted by coupling them to spacetimedependent external sources and adding the resulting expressions to the action. Then, the functional differentiation of the generating functional of the connected Green's functions was performed with respect to these sources [8-10]. It was also shown that taking the identity operator into account explic- 
itly in the action has a particular importance for the renormalization of multilocal composite operators [11].

Recently, there have been made attempts to give a covariant geometric interpretation to the RG flow [12-16]. The space of coupling constants is considered as a differentiable manifold and the RG flow, expressed by means of Lie transport, is interpreted as a particular mapping on it. It has been established by introducing a metric in the coupling constant space that the RG flow is not geodesic in general $[14,15]$. Also the definition of the connection appears problematic [12], although recently a non-metric-compatible definition was given [16].

Our geometric interpretation is less ambitious. We try to implement differential geometric notions along a onedimensional manifold only, the RG trajectory. It is based on parallel transport along the RG trajectory, introduced differently as generally used in the literature. Namely, the space of operators (symbols) is considered as a tangent space at each point of the RG trajectory. This has the advantage that no metric is needed and the connection on the RG trajectory arises in a rather straightforward way, cf. Eq. (4.5) below. It is the geometry for the RG trajectory only that is covered in this manner, ignoring the geometrical aspects of the more complicated space parametrized by the coupling constants.

Nevertheless, our method reveals some advantageous features. The local properties of the RG trajectories, given by the connection, are determined in a unique manner at any point in the coupling constant space. It will be shown that the connection can directly be obtained from the beta functions in terms of the blocked coupling constants. The usual blocking is based on local quantities of the RG flow, such as the beta functions that are sufficient for the local studies in the vicinity of a given fixed point. But the RG flow of more realistic models visits several scaling regimes between the UV and the IR regimes and the determination of the set of important parameters may require global methods that can take into account the interplay between different scaling regimes [17]. The operator renormalization includes in a natural manner a global quantity, called sensitivity matrix, needed for such analysis. A flow equation will be derived for the sensitivity matrix, expressing that the evolution is governed by the connection. This flow equation enables us to perform the renormalization of composite operators beyond the perturbation expansion. In this manner the operator renormalization is better suited for the studies of models with nontrivial IR scaling law than the traditional blocking.

In Sec. II a toy model is presented explaining the origin of operator mixing. Then, the idea of blocking is extended to operators in one-component scalar field theory in Sec. III and in Sec. IV the differential geometric meaning of blocking the operators is clarified. The possibility of finding the scaling operators at an arbitrary scale by means of solving the eigenvalue problem of the sensitivity matrix is discussed in Sec. $\mathrm{V}$. The construction of the blocked operator and the renormalized perturbation expansion (RPE) are compared in Sec. VI. The resolution of the paradoxon of renormalizing irrelevant composite operators in a renormalizable theory is also discussed. The operator mixing matrix is determined for $\phi^{3}$ theory in dimension $d=6$ in a restricted operator basis in the independent mode approximation (IMA) in two different ways in Secs. VII A 1 and VII A 2. The agreement of these results with the one-loop perturbative ones is shown in Sec. VII B. Finally, the main results of the paper are summarized in Sec. VIII.

\section{TOY MODEL FOR OPERATOR MIXING}

Let us consider a zero-dimensional model with two degrees of freedom, $x$ ("the low-frequency one") and $y$ ("the high-frequency one"), and the bare action

$$
S(x, y)=\frac{1}{2} s_{x} x^{2}+\frac{1}{2} s_{y} y^{2}+\sum_{n=0}^{\infty} g_{n}(x+y)^{n}
$$

with the bare couplings $g_{n}$. We are looking for the blocked action $S(x)$ obtained by integrating out the degree of free$\operatorname{dom} y$,

$$
e^{-S(x)}=\int d y e^{-S(x, y)}
$$

The composite operators of the bare theory are defined as $(x+y)^{n}$ with $n=0,1,2, \ldots$. According to relation (2.2), the blocked action $S(x)$ as the function of the bare coupling constants $g_{n}$ can be considered as the generator function for the composite operators for a given value $x$,

$$
\frac{\partial S(x)}{\partial g_{n}}=\frac{\int d y(x+y)^{n} e^{-S(x, y)}}{\int d y e^{-S(x, y)}},
$$

i.e., the partial derivative of the blocked action with respect to one of the bare couplings $g_{n}$ is equal to the "highfrequency" average of the corresponding bare composite operator $(x+y)^{n}$. As a consequence the relation

$$
\frac{\int d x \frac{\partial S(x)}{\partial g_{n}} e^{-S(x)}}{\int d x e^{-S(x)}}=\frac{\int d x d y(x+y)^{n} e^{-S(x, y)}}{\int d x d y e^{-S(x, y)}}
$$

holds, i.e., the partial derivative of the blocked action with respect to the bare coupling $g_{n}$ can be interpreted as the blocked operator that provides the same expectation value in the blocked theory as the bare operator $(x+y)^{n}$ does in the bare theory. Let us introduce the notation $\left\{x^{n}\right\}=\partial S(x) / \partial g_{n}$ for it. The action $S(x)$ can be expanded in terms of the base operators as

$$
S(x)=\frac{1}{2} s_{x} x^{2}+\sum_{m=0}^{\infty} g_{m}^{\prime} x^{m}
$$

with the blocked couplings $g_{m}^{\prime}=g_{m}^{\prime}\left(g_{0}, g_{1}, g_{2}, \ldots\right)(m$ $=0,1,2, \ldots$ ) being functions of the bare couplings. Using this expansion, the blocked operators can be rewritten in the form 


$$
\left\{x^{n}\right\}=\frac{\partial S(x)}{\partial g_{n}}=\sum_{m=0}^{\infty} x^{m} S_{m n}
$$

with the help of the matrix

$$
S_{m n}=\frac{\partial g_{m}^{\prime}}{\partial g_{n}}
$$

Equation (2.6) has the simple interpretation that the blocked operator $\left\{x^{n}\right\}$ obtained from the bare operator $(x+y)^{n}$ by integrating out the degree of freedom $y$ is a linear combination of the base operators $x^{m}$ with the coefficients given by the operator-mixing matrix $S_{m n}$. Thus, the operator with which one can reproduce the vacuum expectation value of a bare-base operator in the blocked theory is the linear combination of all the base operators with arguments projected into the subspace of the degree of freedom $x$ left over.

Above we chose base operators in the bare theory and searched for operators in the blocked theory that reproduce their vacuum expectation values. Also the opposite question can be formulated: we choose a basis of operators in the blocked theory, $x^{n}$ with $n=0,1,2, \ldots$ and search for the operators $\left[(x+y)^{n}\right]$ of the bare theory that reproduce their expectation values, i.e., for which

$$
\frac{\int d x d y\left[(x+y)^{n}\right] e^{-S(x, y)}}{\int d x d y e^{-S(x, y)}}=\frac{\int d x x^{n} e^{-S(x)}}{\int d x e^{-S(x)}}
$$

assuming that the bare theory underlying the blocked one is known. Looking for the bare operators in the general form $\left[(x+y)^{n}\right]=\sum_{m=0}^{\infty} \alpha_{m}^{(n)}(x+y)^{m}$, and using Eqs. (2.4) and (2.6), Eq. (2.8) leads to the relation

$$
\sum_{m=0}^{\infty} x^{n^{\prime}} S_{n^{\prime} m} \alpha_{m}^{(n)}=x^{n}
$$

and one finds that the matrix $\alpha_{m}^{(n)}=\left(S^{-1}\right)_{m n}$ is the inverse of the operator-mixing matrix given by Eq. (2.6). Thus, the bare operators looked for are given by

$$
\left[(x+y)^{n}\right]=\sum_{m=0}^{\infty}(x+y)^{m}\left(S^{-1}\right)_{m n} .
$$

We see again, that the bare operators reproducing the expectation values of given base operators of the blocked theory in the bare one are the linear combinations of the base operators.

It is instructive to generalize the toy model for three variables,

$$
S(x, y, z)=\frac{1}{2} s_{x} x^{2}+\frac{1}{2} s_{y} y^{2}+\frac{1}{2} s_{z} z^{2}+\sum_{n=0}^{\infty} g_{n}(x+y+z)^{n} .
$$

The blocking gives rise the chain of effective theories,

$$
e^{-S(x, y)}=\int d z e^{-S(x, y, z)}, e^{-S(x)}=\int d y e^{-S(x, y)},
$$

and operator mixing

$$
\begin{gathered}
\left\{x^{n}\right\}_{z}=\frac{\partial S(x, y, z)}{\partial g_{n}}=(x+y+z)^{n}, \\
\left\{x^{n}\right\}_{y}=\frac{\partial S(x, y)}{\partial g_{n}}=-\frac{\frac{\partial}{\partial g_{n}} \int d z e^{-S(x, y, z)}}{\int d z e^{-S(x, y, z)}}, \\
\left\{x^{n}\right\}_{x}=\frac{\partial S(x)}{\partial g_{n}}=-\frac{\frac{\partial}{\partial g_{n}} \int d y d z e^{-S(x, y, z)}}{\int d y d z e^{-S(x, y, z)}} .
\end{gathered}
$$

These relations yield

$$
\left\{x^{n}\right\}_{y}=\frac{\int d z\left\{x^{n}\right\}_{z} e^{-S(x, y, z)}}{\int d z e^{-S(x, y, z)}},\left\{x^{n}\right\}_{x}=\frac{\int d y\left\{x^{n}\right\}_{y} e^{-S(x, y)}}{\int d y e^{-S(x, y)}}
$$

indicating that the evolution of the operators comes from the elimination of the field variable in their definition. One can compute the expectation value of $\left\{x^{n}\right\}$ at any level,

$$
\begin{aligned}
\frac{\int d x d y d z\left\{x^{n}\right\}_{z} e^{-S(x, y, z)}}{\int d x d y d z e^{-S(x, y, z)}} & =\frac{\int d x d y\left\{x^{n}\right\}_{y} e^{-S(x, y)}}{\int d x d y e^{-S(x, y)}} \\
& =\frac{\int d x\left\{x^{n}\right\}_{x} e^{-S(x)}}{\int d x e^{-S(x)}} .
\end{aligned}
$$

In other words, the cutoff dependence of the renormalized operators is introduced in such a manner that the expectation values can be recovered for an arbitrary value of the cutoff.

We learned from the toy model discussed above that operator mixing is an immediate consequence of keeping the expectation values unchanged under integrating out degrees of freedom.

\section{OPERATOR MIXING}

Here we define blocked operators in quantum-field theory following the line illustrated by the zero-dimensional toy model of the previous section.

Let us consider the theory given by the bare action 


$$
S_{\Lambda}[\phi]=\int d x \sum_{n} G_{n}(x, \Lambda) O_{n}[\phi(x)],
$$

where $O_{n}[\phi(x)]$ represents a complete set of local operators [function of $\phi(x)$ and its space-time derivatives] coupled to the external sources $G_{n}(x, \Lambda)$. The scalar field $\phi(x)$ $=\varphi_{k}(x)+\chi_{k}(x)$ is decomposed into a low-frequency part

$$
\varphi_{k}(x)=\sum_{|p| \leqslant k} \phi_{p} e^{i p x}
$$

and a high-frequency part

$$
\chi_{k}(x)=\sum_{|p| \in[k, \Lambda]} \phi_{p} e^{i p x}
$$

with the UV cutoff $\Lambda$ and the sharp-moving cutoff $k$. Here inhomogeneous external sources have been introduced for later convenience and assumed that their zero modes $g_{n}(\Lambda)$ are separated,

$$
G_{n}(x, \Lambda)=g_{n}(x, \Lambda)+g_{n}(\Lambda) .
$$

Notice that the variables $n$ and $x$ of the operator $O_{n}[\phi(x)]$ identify a member of the complete set. One can simplify the expressions by introducing a single index $\tilde{n}$ for the pair $(n, x)$ and $\Sigma_{\tilde{n}}$ for the integral and sum $\Sigma_{n} \int d x$, e.g.,

$$
S_{\Lambda}[\phi]=\sum_{\tilde{n}} G_{\tilde{n}}(\Lambda) O_{\tilde{n}}(\phi)
$$

It is required that the partition function of the blocked theory $Z_{k}$ and that of the bare one $Z_{\Lambda}$ are identical:

$$
\frac{\int \mathcal{D} \varphi_{k} \mathcal{D} \chi_{k} e^{-S_{\Lambda}\left[\varphi_{k}+\chi_{k}\right]}}{\left.\int \mathcal{D} \varphi_{k} \mathcal{D} \chi_{k} e^{-S_{\Lambda}\left[\varphi_{k}+\chi_{k}\right]}\right|_{g_{n}(x, \Lambda)=0}}=\frac{\int \mathcal{D} \varphi_{k} e^{-S_{k}\left[\varphi_{k}\right]}}{\left.\int \mathcal{D} \varphi_{k} e^{-S_{k}\left[\varphi_{k}\right]}\right|_{g_{n}(x, k)=0}}
$$

that leads to the definition of the blocked action $S_{k}\left[\varphi_{k}\right]$ up to a constant,

$$
e^{-S_{k}\left[\varphi_{k}\right]}=\int \mathcal{D} \chi_{k} e^{-S_{\Lambda}\left[\varphi_{k}+\chi_{k}\right]}
$$

As shown in [2] the blocked action satisfies the WegnerHoughton equation

$$
\frac{\partial S_{k}\left[\varphi_{k}\right]}{\partial k}=-\lim _{\delta k \rightarrow 0} \frac{1}{2 \delta k} \operatorname{Tr} \ln \frac{\delta^{2} S_{k}}{\delta \varphi_{k} \delta \varphi_{k}}
$$

where the trace is taken on the functional space with Fourier modes $k-\delta k<p<k$. The blocked action can be expanded in the base operators as

$$
S_{k}\left[\varphi_{k}\right]=\sum_{\tilde{n}} G_{\tilde{n}}^{\sim}(k) O_{\tilde{n}}^{\sim}\left(\varphi_{k}\right)
$$

and Eq. (3.8) rewritten in the form of a coupled set of differential equations

$$
k \partial_{k} G_{\tilde{n}}(k)=\beta_{\tilde{n}}(k, G)
$$

for the blocked external sources $G_{\tilde{n}}(k)$. The right-hand sides of these equations represent explicit expressions for the beta functions as functions of the blocked sources.

Let us now turn to the definition of blocked operators. From Eq. (3.7), one gets following functional differentiation

$$
\frac{\delta S_{k}\left[\varphi_{k}\right]}{\delta G_{\tilde{n}}(\Lambda)}=\frac{\int \mathcal{D} \chi_{k} O \tilde{n}\left(\varphi_{k}+\chi_{k}\right) e^{-S_{\Lambda}\left[\varphi_{k}+\chi_{k}\right]}}{\int \mathcal{D} \chi_{k} e^{-S_{\Lambda}\left[\varphi_{k}+\chi_{k}\right]}},
$$

i.e., the functional derivative of the blocked action with respect to any of the bare sources $G_{\tilde{n}}(\Lambda)$ is equal to the highfrequency average of the corresponding bare operator $O \tilde{n}\left(\varphi_{k}+\chi_{k}\right)$. Thus the functional derivative $\delta S_{k}\left[\varphi_{k}\right] / \delta G_{n}(\Lambda)$ reproduces the expectation value of the bare operator $O_{\tilde{n}}\left(\varphi_{k}+\chi_{k}\right)$,

$$
\begin{aligned}
& \int \frac{\mathcal{D} \varphi_{k} \frac{\delta S_{k}\left[\varphi_{k}\right]}{\delta G_{\tilde{n}}(\Lambda)} e^{-S_{k}\left[\varphi_{k}\right]}}{\int \mathcal{D} \varphi_{k} e^{-S_{k}\left[\varphi_{k}\right]}} \\
& =\frac{\int \mathcal{D} \varphi_{k} \mathcal{D} \chi_{k} O \tilde{n}\left(\varphi_{k}+\chi_{k}\right) e^{-S_{\Lambda}\left[\varphi_{k}+\chi_{k}\right]}}{\int \mathcal{D} \varphi_{k} \mathcal{D} \chi_{k} e^{-S_{\Lambda}\left[\varphi_{k}+\chi_{k}\right]}} .
\end{aligned}
$$

As a result the functional derivative of the blocked action with respect to one of the bare external sources, $G_{\tilde{n}}(\Lambda)$ can be interpreted as the operator obtained by blocking from the base operator $O_{\tilde{n}}\left(\varphi_{k}+\chi_{k}\right)$. Let us introduce the notation

$$
\left\{O_{\tilde{n}}^{\sim}\left(\varphi_{k}\right)\right\}_{k}=\frac{\delta S_{k}\left[\varphi_{k}\right]}{\delta G_{\tilde{n}}(\Lambda)}
$$

for the corresponding blocked operator. Making use of expansion (3.9) of the blocked action, the operator mixing

$$
\left\{O_{\tilde{n}}\left(\varphi_{k}\right)\right\}_{k}=\sum_{\tilde{m}} \frac{\delta G_{\tilde{m}}(k)}{\delta G_{\tilde{n}}(\Lambda)} \frac{\delta S_{k}\left[\varphi_{k}\right]}{\delta G_{\tilde{m}}(k)}=\sum_{\tilde{m}} O_{\tilde{m}}\left(\varphi_{k}\right) S_{\tilde{m} \tilde{n}}(k, \Lambda)
$$

can be given in terms of the sensitivity matrix

$$
S_{\tilde{m} \tilde{n}}(k, \Lambda)=\frac{\delta G_{\tilde{m}}(k)}{\delta G_{\tilde{n}}(\Lambda)},
$$

which shows the dependence of the blocked coupling constants on the initial values of the RG flow. As such it is a global feature of the RG trajectory. 
Conversely, if one chooses the base operators $O_{\tilde{n}}^{\sim}\left(\varphi_{k}\right)$ at the scale $k$ then one can search for the bare operator $\left[O_{\tilde{n}}\left(\varphi_{k}+\chi_{k}\right)\right]_{k}$ that reproduces the vacuum expectation value of $O_{\tilde{n}}\left(\varphi_{k}\right)$. An argument similar to that for the toy model in the previous section leads to

$$
\left[O_{\tilde{n}}\left(\varphi_{k}+\chi_{k}\right)\right]_{k}=\sum_{\tilde{m}} O_{\tilde{m}}\left(\varphi_{k}+\chi_{k}\right)\left[S^{-1}(k, \Lambda)\right]_{\tilde{m} \tilde{n}}
$$

Now we pass the RG trajectory in the opposite direction (from small $k$ to large $k$ values) when asking for the bare operator that reproduces the same vacuum expectation value as a given operator at the scale of small $k$. Therefore, the answer is given in terms of the inverse of the matrix $S_{\tilde{m} \tilde{n}}(k, \Lambda)$ describing the mixing of operators if the RG trajectory is passed from large $k$ values toward the small ones as it happened when defining the blocked operators $\left\{O_{\tilde{n}}\left(\varphi_{k}\right)\right\}_{k}$. Notice that the relations (3.14) and (3.16) represent operator equations since Eq. (3.12) remains valid after the insertion of any other operator in the path integral of the numerator at both sides.

It turns out to be useful to derive partial differential equations for the $k$ dependence of the operator-mixing matrix. Using

$$
G_{\tilde{n}}^{\sim}(k-\delta k)=G_{\tilde{n}}^{\sim}(k)-\frac{\delta k}{k} \beta_{\tilde{n}}^{\sim}(k, G)
$$

for an arbitrary infinitesimal change $\delta k$ of the scale $k$, one can write

$$
\begin{aligned}
S_{\tilde{m} \tilde{n}}(k-\delta k) & =\frac{\delta G_{\tilde{m}}(k-\delta k)}{\delta G_{\tilde{n}}^{\tilde{N}(\Lambda)}} \\
& =\sum_{\tilde{l}} \frac{\delta G_{\tilde{m}}(k-\delta k)}{\delta G_{\tilde{l}}(k)} \frac{\delta G_{\tilde{l}}(k)}{\delta G_{\tilde{n}}(\Lambda)} \\
& =\sum_{\tilde{l}}\left[\delta_{\tilde{m} \tilde{l}}-\frac{\delta k}{k} \frac{\delta \beta_{\tilde{m}}(k, G)}{\delta G_{\tilde{l}}(k)}\right] S_{\tilde{l} \tilde{n}}(k) .
\end{aligned}
$$

Subtracting Eq. (3.15), dividing by $\delta k$, and taking the limit $\delta k \rightarrow 0$, we find the set of coupled differential equations for the elements of the operator-mixing matrix:

$$
k \partial_{k} S_{\tilde{m} \tilde{n}}(k, \Lambda)=\sum_{\tilde{l}} \frac{\delta \beta_{\tilde{m}}(k)}{\delta G_{\tilde{l}}(k)} S_{\tilde{l} \tilde{n}}(k, \Lambda) .
$$

The scale dependence of the operator-mixing matrix is governed by the matrix

$$
k \Gamma_{\tilde{n} \tilde{m}}(k)=\frac{\delta \beta_{\tilde{n}}^{\tilde{n}}(k)}{\delta G_{\tilde{m}}(k)}
$$

determining the change of the slope of the RG trajectory due to the variation of the actual point of the parameter space it passes through. The matrix $\Gamma_{\tilde{n} \tilde{m}}(k)$ is a local feature of the $\mathrm{RG}$ trajectory on the contrary to the sensitivity matrix.

\section{PARALLEL TRANSPORT}

Now we show that the operator mixing due to blocking can be interpreted as the parallel transport of operators along the RG trajectory with the connection $\Gamma_{\tilde{n} \tilde{m}}(k)$.

It is the main idea behind operator renormalization that those operators are searched for that reproduce the same vacuum expectation value at different scales,

$$
\begin{array}{r}
\int \mathcal{D} \phi_{k^{\prime}} \mathcal{D} \chi_{k^{\prime}} O \tilde{n}\left(\varphi_{k^{\prime}}+\chi_{k^{\prime}}\right) e^{-S_{k^{\prime}}\left[\varphi_{k^{\prime}}+\chi_{k^{\prime}}\right]} \\
\int \mathcal{D} \phi_{k^{\prime}} \mathcal{D} \chi_{k^{\prime}} e^{-S_{k^{\prime}}\left[\varphi_{k^{\prime}}+\chi_{k^{\prime}}\right]} \\
=\frac{\int \mathcal{D} \phi_{k} \mathcal{D} \chi_{k} O \tilde{n}\left(\varphi_{k}+\chi_{k}\right) e^{-S_{k}\left[\varphi_{k}+\chi_{k}\right]}}{\int \mathcal{D} \phi_{k} \mathcal{D} \chi_{k} e^{-S_{k}\left[\varphi_{k}+\chi_{k}\right]}} .
\end{array}
$$

This relation follows from observing that the right-hand side of Eq. (3.12), the expectation value of a given bare operator of the complete cutoff theory, is independent of the choice of $k$, the way the modes are split into the UV and IR classes. Equation (4.1) allows us to identify the effect of the changing of the cutoff in the renormalized operator,

$$
\left\{O_{\tilde{n}}\left(\varphi_{k^{\prime}}\right)\right\}_{k^{\prime}}=\frac{\int \mathcal{D} \xi\left\{O_{\tilde{n}}\left(\varphi_{k^{\prime}}+\xi\right)\right\}_{k} e^{-S_{k}\left[\varphi_{k^{\prime}}+\xi\right]}}{\int \mathcal{D} \xi e^{-S_{k}\left[\varphi_{k^{\prime}}+\xi\right]}}
$$

for $k^{\prime}<k$. The integration $\mathcal{D} \xi$ extends over the modes with $k^{\prime}<|p|<k$. The operator $\left\{O_{\tilde{n}}\left(\varphi_{k^{\prime}}\right)\right\}_{k^{\prime}}$ is a linear superposition of the base operators $O_{\tilde{n}}\left(\varphi_{k^{\prime}}\right)$ as indicated in Eq. (3.14).

This result suggests a differential geometric interpretation of the operator mixing, its identification with a certain parallel transport. In particular, a $k$-dependent operator $O_{k}$ will be said to be parallel transported in the scale $k$,

$$
O_{k^{\prime}}=P_{k \rightarrow k^{\prime}} O_{k}
$$

if its insertion into any expectation value yields a $k$-independent result, $\partial_{k}\left\langle O_{k}\right\rangle=0$. The mapping $P_{k \rightarrow k^{\prime}}$ of the operators is obviously linear.

Since the operator mixing is linear according to Eq. (3.14), the parallel transport of an operator in the scale, i.e., the parallel-transported operator $O_{k}$, can be characterized by introducing the covariant derivative of the operators,

$$
D_{k} O_{k}=\left(\partial_{k}-\Gamma\right) O_{k}
$$

in such a manner that $D_{k} O_{k}=0$ for the parallel transport. Equation (3.19) suggests the identification of the connection with the matrix $\Gamma$ of Eq. (3.20).

The formal definition of the covariant derivative is the following. The scale dependence in the expectation value $\left\langle O_{k}\right\rangle$ comes from two different sources: the explicit $k$ depen- 
dence of the operator and the implicit $k$ dependence generated by the path integration by taking the expectation value. The operator mixing balances them. We introduce the covariant derivative by the relation

$$
\partial_{k}\left\langle O_{k}\right\rangle=\left\langle D_{k} O_{k}\right\rangle
$$

requiring that the operator mixing generated by the connection amounts to the implicit $k$ dependence of the expectation value. Once the covariant derivative is known the parallel transport can be reconstructed as

$$
P_{k \rightarrow k^{\prime}}=\mathcal{P} \exp ^{k_{k}^{k^{\prime}} d k^{\prime \prime} \Gamma\left(k^{\prime \prime}\right)},
$$

where $\mathcal{P}$ stands for the ordering according to the parameter $k^{\prime \prime}$.

It is obvious that the connection $\Gamma$ is vanishing in the basis $\left\{O_{\tilde{n}}\right\}_{k}$,

$$
\begin{aligned}
\partial_{k}\left\langle\sum_{\tilde{n}} c_{\tilde{n}}(k)\left\{O_{\tilde{n}}\right\}_{k}\right\rangle & =\sum_{\tilde{n}} \partial_{k} c_{\tilde{n}}(k)\left\langle\left\{O_{\tilde{n}}\right\}_{k}\right\rangle \\
& =\left\langle\partial_{k} \sum_{\tilde{n}} c_{\tilde{n}}(k)\left\{O_{\tilde{n}}\right\}_{k}\right\rangle .
\end{aligned}
$$

The connection can in principle be found in any other basis by simple computation. As far as the basis $O_{\tilde{n}}\left(\varphi_{k}\right)$ is concerned it is simpler to check directly that Eq. (4.4) satisfies Eq. (4.5). For this end we insert the arbitrary operator

$$
O_{k}=\sum_{\tilde{m}} c_{\tilde{m}}(k) O_{\tilde{m}}\left(\varphi_{k}\right)=c(k) O
$$

into Eq. (4.5),

$$
\partial_{k}[c(k)\langle\underline{O}\rangle]=\left\langle D_{k} c(k) \underline{O}\right\rangle,
$$

and write, with Eq. (4.4),

$$
\partial_{k} c(k)\langle\underline{O}\rangle+\underline{c}(k) \partial_{k}\left\langle\underline{O O}=\partial_{\underline{k}} c(k)\langle\underline{O O}\rangle-\underline{\langle O}\right\rangle_{k} \Gamma c(k) .
$$

In order to prove Eq. (4.5), we need the relation

$$
-\partial_{k}\langle\underline{\langle O}=\underline{\langle O}\rangle \underline{\Gamma} .
$$

Using $S_{k=0}\left(G_{\tilde{n}}(k)\right)$ as the generator function for $\left\langle O_{\tilde{n}}\right\rangle$, the left-hand side (LHS) of Eq. (4.11) can be written as

$$
\begin{aligned}
& \frac{1}{\delta k}\left[\frac{\delta S_{0}}{\delta G_{\tilde{m}}(k-\delta k)}-\frac{\delta S_{0}}{\delta G_{\tilde{m}}(k)}\right] \\
& \quad=\frac{1}{\delta k}\left[\frac{\delta S_{0}}{\delta G_{\tilde{m}}(k-\delta k)}-\sum_{\tilde{n}} \frac{\delta S_{0}}{\delta G_{\tilde{n}}(k-\delta k)} \frac{\delta G_{\tilde{n}}(k-\delta k)}{\delta G_{\tilde{m}}(k)}\right] \\
& \quad=\frac{1}{\delta k} \sum_{\tilde{n}} \frac{\delta S_{0}}{\delta G_{\tilde{n}}(k-\delta k)}\left[\delta_{\tilde{n} \tilde{m}}-\frac{\delta G_{\tilde{n}}(k-\delta k)}{\delta G_{\tilde{m}}(k)}\right] \\
& \quad=\frac{1}{k} \sum_{\tilde{n}} \frac{\delta S_{0}}{\delta G_{\tilde{n}}(k)} \frac{\delta \beta_{\tilde{n}}(k)}{\delta G_{\tilde{m}}(k)},
\end{aligned}
$$

in the limit $\delta k \rightarrow 0$. According to (3.19), the last line agrees with the right-hand side (RHS) of Eq. (4.11).

Let us turn now to the usual quantum-field theory problem without inhomogeneous external sources, that have only been introduced as technical tools, i.e., take the limit $g_{\tilde{n}}(k)$ $\rightarrow 0$. Then the operator-mixing matrix and the connection reduce to

$$
\begin{gathered}
S_{\tilde{m} \tilde{n}}(k, \Lambda)=\delta\left(x_{m}-x_{n}\right) s_{m n}(k, \Lambda), \\
\Gamma_{\tilde{m} \tilde{n}}(k)=\delta\left(x_{m}-x_{n}\right) \gamma_{m n}(k),
\end{gathered}
$$

respectively, with the coordinate-independent matrices

$$
s_{m n}(k, \Lambda)=\frac{\partial g_{m}(k)}{\partial g_{n}(\Lambda)}, k \gamma_{m n}(k)=\frac{\partial \beta_{m}(k)}{\partial g_{n}(k)},
$$

and the RG equations for the operator-mixing matrix take the form

$$
\partial_{k} s_{m n}(k, \Lambda)=\sum_{l} \gamma_{m l}(k) s_{l n}(k, \Lambda) .
$$

\section{RG FLOW AND UNIVERSALITY}

It has been found that the operator mixing, the problem of keeping the expectation values cutoff independent can be handled by a linear transformation, the parallel transport of the operators. We shall show in this section that the salient features of the RG flow can be recovered from this parallel transport alone.

The scaling combinations of the coupling constants are introduced traditionally in the vicinity of a fixed point $G_{\tilde{m}}^{*}$ in the space of the coupling constants that have been made dimensionless by the help of the cutoff $k$. The basic assumption of the RG strategy is that the evolution equations can be linearized around the fixed points,

$$
\beta_{\tilde{n}} \approx \sum_{\tilde{m}} \Gamma_{\tilde{n} \tilde{m}}^{*}\left(G_{\tilde{m}}^{\tilde{m}}-G_{\tilde{m}}^{*}\right) .
$$

The relevant, marginal, and irrelevant coupling constants are the superpositions 


$$
G_{\tilde{n}}^{s c}=\sum_{\tilde{m}} \bar{c}_{\tilde{n} \tilde{m}}\left(G_{\tilde{m}}-G_{\tilde{m}}^{*}\right)
$$

made by the left eigenvectors of $\Gamma$,

$$
\sum_{\tilde{m}} \bar{c}_{\tilde{n} \tilde{m}} \Gamma_{\tilde{m} \tilde{r}}^{*}=\alpha_{\tilde{n}} \bar{c} \tilde{n} \tilde{r}
$$

with $\alpha_{n}^{\sim}<0, \alpha_{n}^{\sim}=0$ and $\alpha_{n}^{\sim}>0$, respectively. The scale dependence of the scaling coupling constants $G_{\tilde{n}}^{s c}$ is

$$
G_{\tilde{n}}^{s c} \sim k^{\alpha \tilde{n}}
$$

Let us consider a local operator of the form

$$
O[\phi(x)]=\sum_{n} b_{n} O_{n}[\phi(x)]
$$

where $O_{n}[\phi(x)]$ is the product of the terms $\partial_{\mu_{1}} \cdots \partial_{\mu_{l}} \phi^{m}(x)$. It will be necessary to separate for the possible scale-dependent rescaling factors. For this end we introduce the norm

$$
\|O\|=\sqrt{\sum_{n} b_{n}^{2}}
$$

and adopt the convention that the coupling constants $G_{\tilde{n}}^{\sim}(\Lambda)$ of the bare action always multiply local operators with unit norm, $\left\|O_{\tilde{n}}(\Lambda)\right\|=1$. As long as we consider local operators the norm defined above is sufficient and no index with continuous range is needed in its definition. It will be useful to consider the normalized operator flow,

$$
\bar{O}=\frac{O}{\|O\|}
$$

in addition to the original one, $O$.

The scaling operators

$$
O_{\tilde{n}}^{s c}=\sum_{\tilde{m}} c_{\tilde{n} \tilde{m}} O_{\tilde{m}}
$$

are obtained by means of the right eigenvectors of $\Gamma^{*}$,

$$
\sum_{\tilde{m}} \Gamma_{\tilde{r} \tilde{m}}^{*} c_{\tilde{m} \tilde{n}}=\alpha \tilde{r} c_{\tilde{r} \tilde{n}}
$$

satisfying the conditions of completeness $c \cdot \bar{c}=1$ and orthonormality $\bar{c} \cdot c=1$. The coupling constants of the action

$$
S_{k}=\sum_{\tilde{n}} G_{\tilde{n}}(k) \overline{O_{\tilde{n}}^{s c}\left(\phi_{k}\right)}
$$

obviously follow Eq. (5.4). The operator

$$
O=\sum_{\tilde{n}} b_{\tilde{n}} \overline{\left\{O_{\tilde{n}}^{s c}\right\}_{k}}
$$

written at scale $k$ in this basis yields the parallel transport trajectory

$$
\{O\}_{k^{\prime}}=\sum_{\tilde{n}} b_{\tilde{n}}\left\{\overline{\left\{O_{\tilde{n}}^{s c}\right\}_{k}}\right\}_{k^{\prime}}=\sum_{\tilde{n}} b_{\tilde{n}}\left(\frac{k^{\prime}}{k}\right)^{\alpha \tilde{n}} \overline{\left\{O_{\tilde{n}}^{s c}\right\}_{k}}
$$

in the vicinity of the fixed point.

The question we explore now is what information does the flow $\{O\}_{k}$ contain about the importance of certain interactions as the function of the observational scale $k$. Let us start with the remark that according to Eq. (3.13) the fixed point of the blocking relation, where the action, expressed in terms of the dimensionless coupling constants, is scale independent agrees with the fixed point of the operator blocking. The linearization around the fixed points renders the critical exponents of the coupling constants and the scaling operators equivalent since the left and the right spectrum of $\Gamma^{*}$ agree. The operators whose structure converges and changes by an overall factor only are the scaling operators. The corresponding critical exponents can be read off from the evolution of their norm.

The concept of universality stands for the independence of the dimensionless quantities of the long-distance physics from the initial value of the irrelevant coupling constants in the UV scaling regime. The operator $\left\{O_{\tilde{n}}\left(\varphi_{k}\right)\right\}_{k}$ constructed from the local terms of the bare action by parallel transport represents the influence of the bare coupling constants on the physics of the scale $k$. Since the action is dimensionless only relevant operators have nonvanishing parallel transport flow at finite scale according to Eq. (3.13), the parallel transport of the irrelevant operators that are made dimensionless by the running scale $k$ vanishes. Naturally, this does not mean that the effective theories are renormalizable since a renormalizable (relevant) operator parallel transported down from the cutoff mixes with nonrenormalizable (irrelevant) ones, as well.

The operator flow is particularly well suited for the studies of models whose RG flow visits different scaling regimes. In general, any renormalizable theory without manifest scale invariance possesses at least two scaling regimes, one around the UV and another one at the IR fixed points that are separated by a crossover at the intrinsic scale of the theory, $k=k_{c r}$. For models with mass gap the IR scaling regime is trivial, i.e., the relevant operators are Gaussian. Imagine a model with dynamically generated intrinsic scale, e.g., with spontaneous symmetry breaking or condensation or dimensional transmutation, where the IR instability generates nontrivial scaling laws and non-Gaussian relevant coupling constants appear in the IR scaling. Let us suppose that there is a nonrenormalizable operator $O$ that becomes relevant in the IR regime and consider its parallel transport globally, from the UV to the IR fixed point [17]. Since $O$ is nonrenormalizable, $\left\|\{O\}_{k}\right\|$ decreases as $k$ is lowered in the UV reflecting the diminishing importance of a nonrenormalizable operator well below the cutoff. But after having crossed the crossover the flow reflects the properties of a relevant operator, i.e., $\left\|\{O\}_{k}\right\|$ increases as $k$ is further lowered in the IR scaling regime. Now it becomes a competition 
between the UV suppression and the IR enhancement to form the final sensitivity on the cutoff scale parameter. Since the IR increase of the norm is usually fed by IR or collinear divergences, the length of the scaling regimes are determined by $\Lambda / k_{c r}$ and $L k_{c r}$ where $L$ is the size of the system, an IR cutoff. If the coherence is not lost for sufficiently large distances then the condition $\left\|\{O\}_{k}\right\|=1$ can be reached at low enough $k$ for any value of the UV cutoff, i.e., the IR instability can make the otherwise weak sensitivity on the shortdistance physics strong.

Finally, a few words are in order about the possibility of determining the sensitivity matrix. There is a direct method by solving the set (3.19). Namely, the right-hand sides of Eq. (3.10) are analytic expressions for the beta functions that can be differentiated analytically, and the numerical values of the connection matrix then easily computed and used as input for Eq. (3.19). There is also an indirect method according to the definition (3.15). One has to solve the Wegner-Houghton equations (3.10) for the blocked sources as functionals of their initial values at the scale $\Lambda$, and differentiate the solution with respect to these initial conditions. This latter method was used in Ref. [17] to show that the $\phi^{4}$ model in the phase with spontaneously broken symmetry does in fact possess a nonrenormalizable relevant operator in the IR scaling regime.

\section{RG AND RENORMALIZED PERTURBATION EXPANSION}

The comparison of the renormalization of the composite operators presented above with the usual operator mixing obtained in the framework of the renormalized perturbation expansion serves two goals. First, it shows that the usual operator mixing represents the evolution of the composite operators toward the UV direction. Second, it helps to understand an apparent paradox, namely that nonrenormalizable operators can be "renormalized" within a renormalizable theory. The composite operator renormalization stands for the program of finding the counterterms which renders the Green's functions even with the given composite operator insertions finite as the cutoff is removed. Since the finiteness of the Green's functions implies the finiteness of the partition functions where the composite operators are introduced with a source term in the action, as in Eq. (3.9), the completion of this program would amount to the renormalization of theories where the composite operators appear in the action.

\section{A. Renormalized perturbation expansion}

Let us start with the bare action (3.1),

$$
S\left[\phi, G_{\tilde{n}}\right]=\sum_{\tilde{n}} G_{\tilde{n}} O_{\tilde{n}}(\phi) .
$$

For the sake of simplicity, we neglected the subscript $\Lambda$ here, but the dependence on the external sources $G_{\tilde{n}}$ is made explicit; $O_{\tilde{n}}(\phi)$ is a complete set of normalized bare operators. The UV and IR momentum cutoffs, $\Lambda$ and $k$ are assumed to be introduced for the field variable $\phi(x)$ in order to achieve a better comparison with the RG method. Let us separate the zero modes of the sources, i.e., the bare coupling constants $g_{n}, \quad G_{\tilde{n}}=g_{n}+g_{\tilde{n}}$. Furthermore, specify $O_{(1 \mid 1)}[\phi(x)]$ $=-\frac{1}{2} \phi(x) \square \phi(x), O_{1}[\phi(x)]=\phi(x), g_{(1 \mid 1)}=Z_{\phi}, g_{1}=0$. Then, $G_{\tilde{1}}=g_{\tilde{1}}=-j(x)$ is the external current coupled to the bare field.

The corresponding quantum-field theory is defined by the generating functional

$$
Z\left[G_{\tilde{n}}\right]=\frac{\int \mathcal{D} \phi e^{-S\left[\phi, G_{\tilde{n}}\right]}}{\int \mathcal{D} \phi e^{-S\left[\phi ; g_{n}\right]}} .
$$

Then, we obtain the generating functionals $W\left[G_{\tilde{n}}\right]$ $=\ln Z\left[G_{\tilde{n}}^{\tilde{n}}\right]$ and $\Gamma\left[\varphi, G_{\tilde{n}, n \neq 1}\right]=-W\left[G_{\tilde{n}}^{\tilde{n}}\right]+\int d x j(x) \varphi(x)$ of the connected and one-particle irreducible (1PI) Green's functions, respectively, with $\varphi(x)=\delta W / \delta j(x)$.

The insertion of the operator $O_{\tilde{n}}(\phi)$ in the 1PI Green's functions is obtained via functional derivation with respect to the corresponding source $G_{\tilde{n}}$, and the identities

$$
\left\langle O_{\tilde{n}}(\phi)\right\rangle_{1 P I}=-\left.\frac{1}{Z} \frac{\delta Z}{\delta G_{\tilde{n}}}\right|_{0}=-\left.\frac{\delta W}{\delta G_{\tilde{n}}}\right|_{0}=\frac{\delta \Gamma}{\delta G_{\tilde{n}}} 0
$$

hold for $n \neq 1$. The subscript $\left.\right|_{0}$ stands for $g_{\tilde{n}} \equiv 0$ (and $\varphi$ $=0$ for $\Gamma$ ). Owing to its definition via the bare action, this vacuum expectation value is expressed in terms of the bare couplings.

The theory defined above is nonrenormalizable. One has to satisfy infinitely many renormalization conditions in order to fix the renormalized values $g_{n R}$ of the infinitely many coupling constants. This is equivalent to specifying a particular RG trajectory in the RG approach. The theory with nonrenormalizable coupling constants does not allow the removal of the cutoff, i.e., the extrapolation toward short distances is problematic. But as long as the properties far away from the cutoff toward the IR direction are concerned and the possible nontrivial effects of the IR scaling regime [17] are neglected, the values of the nonrenormalizable coupling constants at the cutoff effect the overall scale of the theory only. When considering dimensionless quantities this scale drops out and the nonrenormalizable coupling constants can be set at the cutoff in an arbitrary manner. As a result, there is no problem to construct the operator mixing below a sufficiently high cutoff. One should bear in mind that even the renormalizable theories contain nonrenormalizable operators, the regulator. In fact, the comparison of a theory with different regularizations shows that the regulators amount to a set of irrelevant operators when written in the action. These regulator terms have tree-level fine-tuning which, according to the universality, is sufficient to keep the cutoff independent dynamics fixed. In what follows we take the usual point of view and the regulators will not be represented in the action.

The renormalization conditions enable one to rewrite the bare action as the sum of the renormalized terms and that of infinitely many counterterms 


$$
S\left[\phi, G_{\tilde{n}}\right]=\sum_{\tilde{n}}\left(G_{\tilde{n} R}+c_{\tilde{n}}\left[G_{\tilde{m} R}\right]\right) O_{\tilde{n}}(\phi) \equiv S_{R}\left[\phi, G_{\tilde{n} R}\right]
$$

in the framework of the RPE. Here we introduced the renormalized sources via $G_{\tilde{n}}=G_{\tilde{n} R}+c_{\tilde{n}}\left[G_{\tilde{m} R}\right]$. As mentioned above, all but finite counterterms influence an overall scale factor only of the theory according to the universality. The cutoff will be kept arbitrarily large but finite and fixed. Below, the notation $c_{(1 \mid 1)}\left[G_{\tilde{m} R}\right]=Z_{\phi}-1$ and the renormalization condition $G_{(1 \mid 1) R} \equiv 1$ are used. The coefficients of the counterterms $c_{\tilde{n}}$ are functionals of the renormalized sources $G_{\tilde{m} R}$. Without loss of generality we can assume that all external sources are cutoff at some momentum $\Lambda_{s} \ll \Lambda$. Then, the counterterms remain local similarly to the case with constant external sources [6] and the coefficients $c_{n}$ are only affected by the zero modes of the sources, i.e., they are independent of the spacetime coordinate $x$ and are functions of the coupling constants $g_{n R}$ and the UV and IR cutoffs, $\Lambda$ and $k$, respectively. Then, the relations $g_{n R}+c_{n}\left(g_{m R}\right)=g_{n}$ and $g_{\tilde{n} R} \equiv g_{\tilde{n}}$ hold. For $n=1$ these yield $-g_{\tilde{1}} \equiv j(x)=j_{R}(x)$ and $g_{1}=c_{1}$ for the renormalization condition $g_{1 R}=0$. We see that $g_{\tilde{n}} \equiv 0$ implies $g_{\tilde{n} R} \equiv 0$ and vice versa.

The renormalized generating functional $Z_{R}$ considered as the functional of the renormalized sources,

$$
Z_{R}\left[G_{\tilde{n} R}\right]=\frac{\int \mathcal{D} \phi(x) e^{-S_{R}\left[\phi, G_{n R}\right]}}{\int \mathcal{D} \phi(x) e^{-S_{R}\left[\phi ; g_{n R}\right]}}=Z\left[G_{\tilde{n}}\right]
$$

is the generating functional of the Green's functions with renormalized-composite-operator insertions. The following equations hold:

$$
W_{R}\left[G_{\tilde{n} R}\right]=\ln Z_{R}\left[G_{\tilde{n} R}\right]=W\left[G_{\tilde{n}}\right],
$$

and

$$
\begin{aligned}
\Gamma_{R}\left[\varphi, G_{\tilde{n} \neq \tilde{1}, R}\right] & =-W_{R}\left[G_{\tilde{n} R}\right]+\int d x j_{R}(x) \varphi(x) \\
& =-W\left[G_{\tilde{n}}\right]+\int d x j(x) \varphi(x) \\
& =\Gamma\left[\varphi, G_{\tilde{n} \neq \tilde{1}}\right],
\end{aligned}
$$

where $\varphi(x)=\delta W_{R} / \delta j_{R}(x)=\delta W / \delta j(x)$.

The renormalized-composite-operator insertion $\left[O_{\tilde{n}}(\phi)\right]_{R}$ $(n \neq 1)$ is obtained by functional derivation with respect to the renormalized external source $G_{\tilde{n} R}$ :

$$
\begin{aligned}
\left\langle\left[O_{\tilde{n}}(\phi)\right]_{R}\right\rangle_{1 P I} & =-\left.\frac{1}{Z_{R}} \frac{\delta Z_{R}\left[G_{\tilde{m} R}\right]}{\delta G_{\tilde{n} R}}\right|_{0} \\
& =-\left.\frac{\delta W_{R}\left[G_{\tilde{m} R}\right]}{\delta G_{\tilde{n} R}}\right|_{0}=\left.\frac{\delta \Gamma_{R}\left[\varphi, G_{\tilde{m} \neq \tilde{1}, R}\right]}{\delta G_{\tilde{n} R}}\right|_{0}
\end{aligned}
$$

Now we find the following relations:

$$
\begin{aligned}
\left.\frac{\delta \Gamma_{R}\left[\varphi, G_{\tilde{m} \neq \tilde{1}, R}\right]}{\delta G_{\tilde{n} R}}\right|_{0} & =\left.\frac{\delta \Gamma\left[\varphi, G_{\tilde{m} \neq \tilde{1}]}\right]}{\delta G_{\tilde{n} R}}\right|_{0} \\
& =\left.\left.\sum_{\tilde{r} \neq \tilde{1}} \frac{\delta G_{\tilde{r}}}{\delta G_{\tilde{n} R}}\right|_{0} \frac{\delta \Gamma\left[\varphi, G_{\tilde{m} \neq \tilde{1}}\right]}{\delta G_{\tilde{r}}}\right|_{0}
\end{aligned}
$$

Making use of the derivative

$$
\left.\frac{\delta G_{\tilde{m}}}{\delta G_{\tilde{n} R}}\right|_{0}=\left(Z^{-1}\right)_{m n} \delta(x-y)
$$

with the operator-mixing matrix

$$
\left(Z^{-1}\right)_{m n}=\delta_{m n}+\frac{\partial c_{m}\left(g_{r R}\right)}{\partial g_{n R}}
$$

(for $n, m \neq 1$ ), one finds

$$
\left[O_{\tilde{n}}(\phi)\right]_{R}=\sum_{m \neq 1} O_{\tilde{m}}(\phi)\left(Z^{-1}\right)_{m n}
$$

for $n \neq 1$. This can be extended to $n, m=1$ by defining $\left(Z^{-1}\right)_{1 m}=\delta_{m 1},\left(Z^{-1}\right)_{n 1}=\delta_{1 n}$. It is worthwhile mentioning that the operator-mixing matrix turned out local in spacetime coordinates, i.e., it is independent of the momentum at which the composite-operator insertion is taken. The matrix $Z_{n m}$ is just the transposed one of that used in Ref. [6].

\section{B. Comparison of the two schemes}

We compare now the notion of renormalized operator in the perturbative approach with the notion of blocked operator in the RG framework. Table I summarizes the formal similarities between the two approaches.

The RG approach keeps the UV cutoff $\Lambda$ fixed and uses a decreasing IR cutoff $k$, so that the RG trajectories are passed toward the IR limit $k \rightarrow 0$. On the contrary, the couplings are defined at some low-energy scale $k=\mu \ll \Lambda$ and the UV cutoff is shifted toward infinity in the RPE, and the RG trajectories are followed just in the opposite direction, toward large momenta. The RG approach reproduces the perturbative results for the ordinary Green's functions in the UV scaling regime [6,7] up to powers and logarithms of $\mu / \Lambda$ that are vanishing in the asymptotic limit $\Lambda \rightarrow \infty$.

It is easy to see that

$$
\sum_{\tilde{n}} G_{\tilde{n}}(\Lambda)\left\{O_{\tilde{n}}\right\}_{k}=\sum_{\tilde{n}} G_{\tilde{n}}(\Lambda) \frac{\delta S_{k}\left[\phi_{k}\right]}{\delta G_{\tilde{n}}(k)}
$$

gives the blocked action with cutoff $k$ in the order $\mathcal{O}[G(\Lambda)]$, the blocking of the action and the operators agree at the linearized level, i.e., they share the fixed points and the critical exponents. The Legendre-transformed effective action with the IR cutoff $k \neq 0[18,21]$ describes the effective theory 
TABLE I. Comparison of various notions in the renormalization group (RG) method and the renormalized perturbation expansion (RPE).

RG

RPE
Coupling at UV scale: $g_{n}(\Lambda)$

Blocked coupling: $g_{n}(k)$

Operator-mixing matrix:

$s_{n m}(k, \Lambda)$

Defined as

$s_{n m}=\frac{\partial g_{n}(k)}{\partial g_{m}(\Lambda)}$

Operator at UV scale: $O \tilde{n}(\phi)$

Blocked operator:

$\left\{O_{\tilde{n}}^{-(}\left(\varphi_{k}\right)\right\}_{k}=\left.\frac{\delta S_{k}}{\delta G_{\tilde{n}}^{-}(\Lambda)}\right|_{g \tilde{m}(\Lambda)=0}$

Operator mixing:

$\left\{O_{\tilde{n}}\left(\varphi_{k}\right)\right\}_{k}=\Sigma_{m} O_{\tilde{m}}\left(\varphi_{k}\right) s_{m n}(k)$

Operator obtained by

inverse blocking

(if exists):

$\left[O_{\tilde{n}}(\phi)\right]_{k}=\Sigma_{m} O_{\tilde{m}}(\phi)\left(s^{-1}\right)_{m n}(k, \Lambda)$
Bare coupling: $g_{n}$

Renormalized coupling: $g_{n R}$

Operator-mixing matrix: $Z_{n m}$

Defined via

$\left(Z^{-1}\right)_{m n}=\frac{\partial g_{m}}{\partial g_{n R}}$

Bare operator: $O_{\tilde{n}}(\phi)$

Not used

Renormalized operator:

$\left[O_{\tilde{n}}(\phi)\right]_{R}=\Sigma_{m} O_{\tilde{m}}(\phi)$

$\times\left(Z^{-1}\right)_{m n}$ after the high-frequency modes have been eliminated, similarly to the blocked action for the low-frequency modes.

The RPE deals with the effective action in the limit $k$ $\rightarrow 0, \Lambda \rightarrow \infty$. Since the bare couplings $g_{n}$ and the renormalized couplings $g_{n R}$ are the analogs of $g_{n}(\Lambda)$ at the UV cutoff scale and of the blocked couplings $g_{n}(k)$, respectively, the operator-mixing matrix $s_{n m}=\partial g_{n}(k) / \partial g_{m}(\Lambda)$ defined in the $\mathrm{RG}$ approach is just the analog of the matrix $Z_{n m}$ defined via $\left(Z^{-1}\right)_{m n}=\partial g_{m} / \partial g_{n R}$ in the RPE. This analogy will be demonstrated in Sec. VII on the equivalence of the one-loop perturbative results with those obtained by the RG method in IMA for a few elements of the operator-mixing matrix in the particular case of $\phi^{3}$ theory in dimension $d=6$.

The analogs of the blocked operators $\left\{O_{\tilde{n}}\left(\varphi_{k}\right)\right\}_{k}$ are not used in the RPE. In the latter one seeks the operator at the scale of the UV cutoff that reproduces the expectation value of an operator given at the renormalization scale. The renormalized operator $[O \tilde{n}(\phi)]_{R}$ satisfying this requirement is the analog of the operator $\left[O_{\tilde{n}}\right]_{k}(\phi)$ introduced in the RG approach by means of inverse blocking.

It is the basic advantage of the RG approach that a non- perturbative answer can be obtained with its help on operator mixing, whereas also the UV finite pieces of the operatormixing matrix are automatically determined. Furthermore, the RG approach enables one to get a deeper insight into the reason of operator mixing as a natural consequence of reproducing the same vacuum expectation values in the bare theory and in the blocked one, or in other words as a direct consequence of integrating out degrees of freedom.

\section{OPERATOR MIXING IN $\phi_{6}^{3}$ THEORY}

The relation between the composite-operator renormalization in the RG scheme and the operator mixing of the RPE is demonstrated in this section in the case of a simple scalar model.

\section{A. RG method}

We start with the determination of the blocked operators in the framework of the $\phi^{3}$ theory in dimension $d=6$ in the independent mode approximation of the next-to-leading order of the derivative expansion and show that both the direct and the indirect methods lead to the same results.

Let us include as base operators $O_{\tilde{n}}^{\sim}\left(\varphi_{k}\right)$ the derivative operators

$$
\begin{aligned}
& D_{(0 \mid 1)}\left(\varphi_{k}\right)=-\square \varphi_{k}, D_{(1 \mid 1)}\left(\varphi_{k}\right)=-\frac{1}{2} \varphi_{k} \square \varphi_{k}, \\
& D_{(0 \mid 2)}\left(\varphi_{k}\right)=-\frac{1}{2} \square \varphi_{k}^{2}
\end{aligned}
$$

and the local potential

$$
V\left(\varphi_{k}\right)=\sum_{l=0}^{\infty} g_{l}(k) \frac{\varphi_{k}^{l}}{l !}
$$

into the ansatz for the blocked action:

$$
\begin{aligned}
S_{k}= & \int d^{d} x\left[Z_{(0 \mid 1)}(k) D_{(0 \mid 1)}\left(\varphi_{k}\right)+Z_{(1 \mid 1)}(k) D_{(1 \mid 1)}\left(\varphi_{k}\right)\right. \\
& \left.+Z_{(0 \mid 2)}(k) D_{(0 \mid 2)}\left(\varphi_{k}\right)+V\left(\varphi_{k}\right)\right] .
\end{aligned}
$$

The bare $\phi^{3}$ theory is specified by the bare couplings $g_{2}(\Lambda)=m^{2}, \quad g_{3}(\Lambda)=\lambda, \quad g_{l \geqslant 4}(\Lambda)=0, \quad Z_{(0 \mid 1)}(\Lambda)=0$, $Z_{(1 \mid 1)}(\Lambda)=1$, and $Z_{(0 \mid 2)}(\Lambda)=-\frac{1}{2}$.

The choice of operators in Eq. (7.3) means that the field dependence of the wave-function renormalization is not taken into account. Terms with higher-order derivatives of the field are also neglected, but the extension of the operator basis is straightforward. Other operators of the discussed types can be expressed as linear combinations of those included in the basis, e.g.,

$$
-\partial_{\mu} \varphi_{k} \cdot \partial_{\mu} \varphi_{k}=D_{(0 \mid 2)}\left(\varphi_{k}\right)-2 D_{(1 \mid 1)}\left(\varphi_{k}\right)
$$

Having derived the explicit forms of the right-hand sides of Eqs. (3.8) and (3.19), the limit $g_{\tilde{n}}(k) \rightarrow 0$ can already be taken at the beginning of the calculation except for the couplings multiplying the operators $\square \varphi_{k}(x)$ and $\square \varphi_{k}^{2}(x)$. The 
TABLE II. Orders of magnitude of the operator-mixing coefficients $s_{n m}$ in IMA for $\lambda \phi^{3}$ theory for $k$ $=0, \Lambda^{2} \gg m^{2} . E(\Lambda)$ indicates a constant plus logarithmically divergent one-loop contributions; $M_{n}$ $=\varphi^{n} / n ! ; d_{n}, d_{m}$ stand for the momentum dimensions of the operators $\left\{O_{n}\right\}, O_{\tilde{m}}$, respectively.

\begin{tabular}{|c|c|c|c|c|c|c|c|c|c|c|}
\hline \multirow[b]{2}{*}{$d_{n}$} & \multicolumn{2}{|c|}{$d_{m}$} & \multirow{2}{*}{$\begin{array}{c}0 \\
M_{0}\end{array}$} & \multirow{2}{*}{$\begin{array}{c}2 \\
M_{1} \\
\end{array}$} & \multirow{2}{*}{${ }^{4} M_{2}$} & \multicolumn{2}{|c|}{6} & \multirow[b]{2}{*}{$D_{(0 \mid 2)}$} & \multirow[b]{2}{*}{$D_{(1 \mid 1)}$} & \multirow{2}{*}{$\begin{array}{c}8 \\
M_{4}\end{array}$} \\
\hline & $\left\{O_{\tilde{n}}\right\}$ & $O_{\tilde{m}}$ & & & & $D_{(0 \mid 1)}$ & $M_{3}$ & & & \\
\hline 0 & & & 1 & 0 & $\Lambda^{4}$ & 0 & 0 & 0 & $\Lambda^{6}$ & 0 \\
\hline 2 & & & 0 & 1 & $\Lambda^{2}$ & 0 & $\Lambda^{4}$ & 0 & $\Lambda^{4}$ & 0 \\
\hline \multirow[t]{2}{*}{4} & & & 0 & 0 & $E(\Lambda)$ & 0 & $\Lambda^{2}$ & 0 & $\Lambda^{2}$ & $\Lambda^{4}$ \\
\hline & & & 0 & 0 & $\ln \Lambda$ & 1 & $\Lambda^{2}$ & $\Lambda^{2}$ & $\Lambda^{2}$ & 0 \\
\hline \multirow[t]{3}{*}{6} & & & 0 & 0 & $\Lambda^{0}$ & 0 & $E(\Lambda)$ & 0 & $\ln \Lambda$ & $\Lambda^{2}$ \\
\hline & & & 0 & 0 & $\Lambda^{0}$ & 0 & $\ln \Lambda$ & $E(\Lambda)$ & $\ln \Lambda$ & $\Lambda^{2}$ \\
\hline & & & 0 & 0 & $\Lambda^{0}$ & 0 & $\ln \Lambda$ & 0 & $E(\Lambda)$ & 0 \\
\hline 8 & & & 0 & 0 & $\Lambda^{0}$ & 0 & $\Lambda^{0}$ & 0 & $\Lambda^{0}$ & $E(\Lambda)$ \\
\hline
\end{tabular}

corresponding terms in the action would yield pure surface terms and therefore, their effects on operator mixing can only be kept track of if the corresponding inhomogeneous sources are replaced by constants only at the end of the calculation.

A further remark is in order here. Namely, we have formulated the whole procedure in the Wegner-Houghton framework with a sharp cutoff and used derivative expansion. It is, however, well known that the sharp cutoff introduces undesirable singularities due to the derivatives of the steplike cutoff function [20]. This may also cause our method in its present form with sharp cutoff to fail in correctly describing the renormalization of the derivative operators. We do not see, however, any objections to reformulate our method of treating composite-operator renormalization using a smooth cutoff on the base of Polchinski's equation [7].

\section{Indirect method}

For the indirect determination of the operator-mixing matrix the following steps should be performed.

(1) Determination of the blocked couplings. Our method to establish the coupled set of differential equations (3.10) for the couplings from Eq. (3.8) is similar to that used in [19]. Namely, we substitute $\varphi_{k}(x)=\varphi_{0}+\eta(x)$ where $\varphi_{0}$ is an arbitrary constant, expand both sides of Eq. (3.8) in Taylor series with respect to the inhomogeneous piece $\eta(x)$, and compare the coefficients of the corresponding operators $O \tilde{n}(\eta)$. Since only operators with second derivatives are considered, it is sufficient to terminate the expansion at the quadratic terms. In Appendix A, the set of coupled first-order differential equations (A11), (A15), (A16), and (A24) is obtained for the blocked couplings. These equations are then solved in independent-mode approximation analytically in Appendix B.

(2) Determination of the operator-mixing matrix in IMA through differentiating the solutions for the blocked couplings with respect to the initial values of the various couplings at the scale $\Lambda$. This step is discussed in detail in Appendix B. The orders of magnitude of the elements of the operator-mixing matrix with respect to the UV cutoff $\Lambda$ are indicated in Table II.

\section{Direct method}

As byproducts, the right-hand sides of Eqs. (A11), (A15), (A16), and (A24) provide us the exact analytic expressions for the beta functions, $\beta_{n}(k)$ (see the beginning of Appendix $C)$. Thus, analytic expressions can be obtained for the elements of the connection $\gamma_{n m}$ by differentiating the appropriate beta functions with respect to the appropriate coupling constants without any approximation. The results are summarized in Appendix $\mathrm{C}$ and the nontrivial matrix elements indicated in Table III. This matrix is the input for Eqs. (3.19)

TABLE III. Elements of the connection matrix $\gamma_{n m}(k)$ for a theory with polynomial potential; the nontrivial matrix elements are indicated by $\times, d_{n}, d_{m}$ denote the momentum dimensions of the base operators $O_{\tilde{n}}(\phi), O_{\tilde{m}}(\phi)$, respectively.

\begin{tabular}{cccccccccc}
\hline \hline & $d_{m}$ & 0 & 2 & 4 & & 6 & & & 8 \\
$d_{n}$ & $n \quad{ }^{2} m$ & 0 & 1 & 2 & $(0 \mid 1)$ & 3 & $(0 \mid 2)$ & $(1 \mid 1)$ & 4 \\
\hline 0 & 0 & 0 & 0 & $\times$ & 0 & 0 & 0 & $\times$ & 0 \\
2 & 1 & 0 & 0 & $\times$ & 0 & $\times$ & 0 & $\times$ & 0 \\
4 & 2 & 0 & 0 & $\times$ & 0 & $\times$ & 0 & $\times$ & $\times$ \\
& $(0 \mid 1)$ & 0 & 0 & $\times$ & 0 & $\times$ & $\times$ & $\times$ & 0 \\
6 & 3 & 0 & 0 & $\times$ & 0 & $\times$ & 0 & $\times$ & $\times$ \\
& $(0 \mid 2)$ & 0 & 0 & $\times$ & 0 & $\times$ & $\times$ & $\times$ & $\times$ \\
& $(1 \mid 1)$ & 0 & 0 & $\times$ & 0 & $\times$ & 0 & $\times$ & 0 \\
8 & 4 & 0 & 0 & $\times$ & 0 & $\times$ & 0 & $\times$ & $\times$ \\
\hline \hline
\end{tabular}


to the direct determination of the operator mixing matrix.

Equations (3.19) can be rewritten as integral equations in a rather compact form introducing the coloumn vectors $s_{n}$ with the elements $s_{m n}$ (with the row index $m$ ) and the connection matrix $\underline{\underline{\gamma}}$ (with the elements $\gamma_{l m}$ ):

$$
\underline{s}_{n}(k)=\underline{s}_{n}(\Lambda)-\int_{k}^{\Lambda} d \kappa \underline{\gamma}(\kappa) \underline{s}_{n}(\kappa) .
$$

This means that all the coloumn vectors $s_{n}(k)$ satisfy the same ordinary first-order linear differential equation; only the initial conditions $s_{n}(\Lambda)$ are different for them. The analytic expressions for the elements of the connection matrix (see Appendix C) should be used as input.

Here we determine the operator-mixing matrix analytically in IMA. For this approximation, all the coupling constants in the explicit expressions for the elements of $\underline{\underline{\gamma}}$ and the coloumn vector $s_{n}(\kappa)$ should be replaced by their $\overline{\bar{r}}$ bare values on the RHS of Eq. (7.5). For the $\lambda \phi^{3}$ theory one has $Z_{(1 \mid 1)}(\Lambda)=1, Z_{(0 \mid 1)}(\Lambda)=0, Z_{(0 \mid 2)}(\Lambda)=-\frac{1}{2}, g_{2}(\Lambda)=m^{2}$, $g_{3}(\Lambda)=\lambda, g_{l \geqslant 4}(\Lambda)=0$. Since there is no operator mixing at the scale $\Lambda$, the initial conditions are $s_{m n}(\Lambda)=\delta_{m n}$. Then, Eqs. (7.5) take the forms

$$
s_{m n}^{I M A}(k)=\delta_{m n}-\int_{k}^{\Lambda} d \kappa \gamma_{m n}^{I M A}(\kappa),
$$

and their solutions can be expressed in terms of the integrals in Appendix E. Using the results of Appendix C, it has been checked that the solutions are just the operator-mixing coefficients found in Appendix B by the indirect method previously.

\section{B. Perturbative approach}

In Appendix $\mathrm{D}$ we determine perturbatively the operatormixing coefficients $\left(Z^{-1}\right)_{n m}$ for $\phi^{3}$ theory in dimension $d$ $=6$ in one-loop approximation. The inverse of $\left(Z^{-1}\right)_{n m}$ can directly be compared with the results obtained for the matrix $s_{m n}$ by means of the RG approach in IMA in Sec. VII A. The inversion of the matrix results in the change of the sign of the terms of $o(\hbar)$ of the matrix elements. Expressing the perturbative results in terms of the loop integrals given in Appendix E, it is easy to recognize that $Z_{n m}=s_{n m}(k=0)$ in the above-mentioned approximations. This agreement illustrates how the perturbative approach is related to the RG approach, that was discussed in Sec. VI B.

\section{SUMMARY}

In the toy model of a zero-dimensional-field theory the operator mixing has been explained as the natural consequence of integrating out degrees of freedom. Then, the notion of blocked operators is defined in one-component scalarfield theory through the requirement of reproducing the same vacuum expectation value in the bare theory and in the blocked (effective) one. The blocking procedure proposed by Wegner and Houghton has been used, i.e., the highfrequency degrees of freedom were integrated out in infini- tesimal momentum shells sequentially using a sharp-moving cutoff.

It is shown that the blocking of operators introduced in this paper satisfies the (semi)group property. Differential equations are derived for the elements of the operator-mixing matrix that should be solved simultaneously with the Wegner-Houghton equations. It is found that the blocking of operators corresponds to parallel transporting them along the RG trajectory-a flat, one-dimensional manifold. The scale dependence of the operator-mixing matrix is governed by the connection, showing the changes of the beta functions due to infinitesimal changes of the couplings at a certain scale $k$. It is also shown that the eigenoperators of the connection are the local scaling operators in any scaling regimes. Thus, solving the eigenvalue problem of the connection enables one in principle to detect different scaling regimes and find the corresponding relevant operators.

The limitations are, of course, the validity region of the Wegner-Houghton equation due to the occurrence of a nontrivial saddle point, and the usage of the sharp cutoff together with the gradient expansion. As to the latter technical problem, a generalization of operator blocking to a smooth cutoff approach seems to be possible by including the appropriate cutoff terms into the action. The reformulation of the whole issue in the framework of renormalization in the internal space [21] would solve both of the above-mentioned problems and do also for generalization to gauge theories.

The differential RG approach and the perturbative approach for operator renormalization are compared in detail. It is explained that the renormalized operator (used in perturbative terms) corresponds to choosing an operator at the renormalization scale and looking for an operator at the UV scale (tending to infinity) that reproduces the vacuum expectation value of the chosen operator. On the particular example of $\phi^{3}$ theory in dimension $d=6$ the agreement of the results of the RG approach in IMA with the one-loop perturbative ones has been illustrated for the elements of the operator-mixing matrix in a truncated basis of operators.

The main advantage of the flow equations for the sensitivity matrix presented is that by their help the renormalization of composite operators can be performed beyond the perturbative regime. The method has the power to go beyond the independent mode approximation by solving the flow equations numerically. A comparison of the nonperturbative results with the perturbative ones, e.g., for the well-known $\phi^{3}$ theory would certainly be interesting. Various models with nontrivial IR scaling could be analyzed down to the scale where the IR instabilities responsible for the nontrivial scaling occur.

\section{ACKNOWLEDGMENTS}

One of the authors (K.S.) expresses his gratitude to the Humboldt Foundation for the follow-up grant, to W. Greiner and G. Soff for their kind hospitality, and thanks I. Lovas, Z. Nagy, G. Plunien, and R. Schützhold for the useful discussions. Valuable discussions with N. Sadooghi are also appreciated. This work has been supported by Grant Nos. OTKA T023844/1997, T29927/98, NATO SA(PST.CLG 
975722)5066, DFG-MTA 436UNG113/140/0, and MÖBDAAD 27 (323-PPP-Ungarn).

\section{APPENDIX A: RG EQUATIONS FOR THE BLOCKED COUPLINGS}

The RG equations for the blocked couplings figuring in the blocked action (7.3) are derived by separating the zero mode $\varphi_{0}$ of the field $\varphi_{k}(x), \varphi_{k}(x)=\varphi_{0}+\eta(x)$ and expanding both sides of Eq. (3.8) in Taylor series with respect to $\eta(x)$ terminated at the quadratic terms. The zeroth-, first-, and second-order terms are

$$
\begin{aligned}
\sigma_{0}= & \int d^{d} x V\left(\varphi_{0}\right), \\
\sigma_{1}= & \int d^{d} x\left\{-\left[Z_{(0 \mid 1)}(x, k)\right.\right. \\
& \left.+\varphi_{0} Z_{(0 \mid 2)}(x, k)\right] \square \eta \\
& \left.+V^{(1)}\left(\varphi_{0}\right) \eta(x)\right\}, \\
\sigma_{2}= & \int d^{d} x\left[-Z_{(1 \mid 1)}(k) \frac{1}{2} \eta \square \eta\right. \\
& -Z_{(0 \mid 2)}(x, k) \frac{1}{2} \square \eta^{2} \\
& \left.+\frac{1}{2} V^{(2)}\left(\varphi_{0}\right) \eta^{2}\right] .
\end{aligned}
$$

Furthermore, we need the second derivative of the blocked action, $S_{k}^{(2)}=A+B+C+o\left(\eta^{3}\right)$ with the matrices $A, B$, and $C$ of zeroth, first, and second order of $\eta$ and having the following forms:

$$
A_{p q}=\mathcal{A}\left(p^{2}\right) V_{d} \delta_{p+q}+\chi_{p q}
$$

with the diagonal part

$$
\mathcal{A}\left(p^{2}\right)=V_{\kappa}^{(2)}\left(\varphi_{0}\right)+Z_{(1 \mid 1)} p^{2},
$$

and the off-diagonal piece

$$
\chi_{p q}=\int d^{d} z Z_{(0 \mid 2)}(z)(p+q)^{2} e^{i(p+q) z} \text {; }
$$

furthermore,

$$
B_{p q}=\mathcal{B} \int d z \eta(z) e^{i(p+q) z}
$$

with $\mathcal{B}=V^{(3)}\left(\varphi_{0}\right)$, and

$$
C_{p q}=\frac{1}{2} V^{(4)}\left(\varphi_{0}\right) \int d^{d} z \eta^{2} e^{i(p+q) z} .
$$

Rewriting the logarithm on the RHS of Eq. (3.8) as $\ln (A$ $+B+C+\cdots)=\ln A+\ln \left[1+A^{-1}(B+C+\cdots)\right]$ and expanding the second logarithmic term in Taylor series, $A^{-1} B+A^{-1} C$ $-\frac{1}{2} A^{-1} B A^{-1} B \cdots$, one finds the equations

$$
\begin{aligned}
k \partial_{k} \sigma_{0}= & -k^{d} \alpha_{d} \int \frac{d \omega_{n}}{\Omega_{d}}(\ln A)_{k n,-k n}, \\
k \partial_{k} \sigma_{1}= & -k^{d} \alpha_{d} \int \frac{d \omega_{n}}{\Omega_{d}}\left(A^{-1} B\right)_{k n,-k n}, \\
k \partial_{k} \sigma_{2}= & -k^{d} \alpha_{d} \int \frac{d \omega_{n}}{\Omega_{d}} \\
& \times\left(A^{-1} C-\frac{1}{2} A^{-1} B A^{-1} B\right)_{k n,-k n} .
\end{aligned}
$$

Here, the matrix products of the form $(M N)_{p q}$ $=\Sigma_{P} M_{p,-P} N_{P, q}$ should be understood over a restricted phase space, i.e., the sum over $P$ should be restricted to the thin momentum shell $k<|P| \leqslant k+\delta k$. Such sums are performed in the continuum limit as integrals over the interval $[k-\epsilon, k+\delta k]$ with $\epsilon>0$ infinitesimal and the limits $\epsilon \rightarrow 0$ and $\delta k \rightarrow 0$ are taken at the end. It has been checked that this procedure provides a result for field-independent wave function renormalization $Z_{(1 \mid 1)}$ in IMA that is in agreement with the perturbative one-loop result obtained, e.g., in [6] for $\phi^{3}$ theory in $d=6$ dimension.

$\sigma_{0}$ : In order to find the explicit form of Eq. (A7), we write

$$
\begin{aligned}
(\ln A)_{p,-p}= & \text { const }+\ln \mathcal{A}\left(p^{2}\right)+\frac{1}{V_{d}}\left(\mathcal{A}^{-1} \chi\right)_{p,-p} \\
& -\frac{1}{2 V_{d}^{2}}\left(\mathcal{A}^{-1} \chi \mathcal{A}^{-1} \chi\right)_{p,-p}+o\left(\chi^{3}\right) .
\end{aligned}
$$

Since $\chi$ contains second powers of the momenta, its first, second, etc. powers generate gradient terms of the order $\partial^{2}$, $\partial^{4}$, etc., respectively. In the second order of the gradient expansion we only have to include the term linear in $\chi$, but its diagonal matrix element vanishes, so that we obtain $(\ln A)_{p,-p} \approx \ln \mathcal{A}\left(p^{2}\right)$ and the integro-differential equation

$$
V_{k}\left(\varphi_{0}\right)=V_{\Lambda}\left(\varphi_{0}\right)+\hbar \alpha_{d} \int_{k}^{\Lambda} d \kappa \kappa^{d-1} \ln \mathcal{A}\left(\kappa^{2}\right)
$$

for the blocked potential $V_{k}\left(\varphi_{0}\right)$. Here $\alpha_{d}=\Omega_{d}(2 \pi)^{-d} / 2$, $\Omega_{d}=2 \pi^{d / 2} / \Gamma(d / 2)$ is the entire solid angle in the $d$-dimensional momentum space.

$\sigma_{1}$ : For the evaluation of the RHS of Eq. (A8) we need the inverse of the nondiagonal matrix $A_{p q}$. Expanding it in powers of the off-diagonal matrix $\chi_{p q}$, one finds

$$
\begin{aligned}
\left(A^{-1}\right)_{p q}= & \frac{1}{V_{d}} \mathcal{A}^{-1}\left(p^{2}\right) \delta_{p+q}-\frac{1}{V_{d}^{2}} \mathcal{A}^{-1}\left(p^{2}\right) \chi_{p q} \mathcal{A}^{-1}\left(q^{2}\right) \\
& +o\left(\chi^{2}\right) .
\end{aligned}
$$

The trace of the matrix product 


$$
\begin{aligned}
\left(A^{-1} B\right)_{p q}= & \frac{1}{V_{d}} \mathcal{A}^{-1}\left(p^{2}\right) B_{p q}-\frac{1}{V_{d}^{2}} \mathcal{A}^{-1}\left(p^{2}\right) \\
& \times \int^{\prime} \frac{V_{d} d \omega_{P} P^{d-1} d P}{(2 \pi)^{d}} \chi_{p,-P} \mathcal{A}^{-1}\left(P^{2}\right) B_{P q}
\end{aligned}
$$

can be evaluated by performing the integral over the infinitesimal momentum shell as discussed above. Substituting the result in the RHS of Eq. (A8) and comparing the corresponding terms on both of its sides, one gets

$$
\begin{aligned}
& k \partial_{k} V^{(1)}\left(\varphi_{0}\right)=-\hbar k^{d} \alpha_{d} \mathcal{B} \mathcal{A}^{-1}\left(k^{2}\right), \\
& k \partial_{k} Z_{(0 \mid 1)}(k)=\hbar k^{d} \alpha_{d} \mathcal{B}_{0} \mathcal{A}_{0}^{-2} Z_{(0 \mid 2)}(k), \\
& k \partial_{k} Z_{(0 \mid 2)}(k)=\hbar k^{d} \alpha_{d}\left[\frac{V^{(4)}(0)}{\mathcal{A}_{0}^{2}}-\frac{2 \mathcal{B}^{2}}{\mathcal{A}_{0}^{3}}\right] Z_{(0 \mid 2)}(k) .
\end{aligned}
$$

Here Eq. (A14) is the first derivative of Eq. (A11), $\mathcal{A}_{0}$ $=\left.\mathcal{A}\left(k^{2}\right)\right|_{\varphi_{0}=0}, \mathcal{B}_{0}=\left.\mathcal{B}\right|_{\varphi_{0}=0}$.

$\sigma_{2}$ : The trace of the first matrix on the RHS of Eq. (A9) can be evaluated analogously to that of $A^{-1} B$ :

$$
\begin{aligned}
& -\hbar k^{d} \alpha_{d} \int \frac{d \omega_{n}}{\Omega_{d}} \frac{V^{(4)}\left(\varphi_{0}\right)}{\mathcal{A}\left(k^{2}\right)} \\
& \times \int d^{d} z \frac{1}{2}\left[\eta^{2}(z)+Z_{(0 \mid 2)}(z) \square \eta^{2}(z)\right] .
\end{aligned}
$$

Up to the first order of $\chi_{p q}$, one can write

$$
\begin{aligned}
-\frac{1}{2}( & \left.A^{-1} B A^{-1} B\right)_{p q} \\
= & -\frac{1}{2 V_{d}^{2}} \sum_{P}{ }^{\prime} \frac{B_{p,-P} B_{P q}}{\mathcal{A}\left(p^{2}\right) \mathcal{A}\left(P^{2}\right)} \\
& +\frac{1}{2 V_{d}^{3}} \sum_{P}{ }^{\prime} \sum_{Q}{ }^{\prime} \frac{B_{p,-P} \chi_{P,-}{ }^{B} B_{Q q}}{\mathcal{A}\left(p^{2}\right) \mathcal{A}\left(P^{2}\right) \mathcal{A}\left(Q^{2}\right)} \\
& +\frac{1}{2 V_{d}^{3}} \sum_{P}{ }^{\prime} \sum_{Q}{ }^{\prime} \frac{\chi_{p,-Q} B_{Q,-P} B_{P q}}{\mathcal{A}\left(p^{2}\right) \mathcal{A}\left(P^{2}\right) \mathcal{A}\left(Q^{2}\right)}
\end{aligned}
$$

The second and third terms give identical contributions to the RHS of Eq. (A9),

$$
\hbar k^{d} \alpha_{d} \frac{1}{2} \int \frac{d \omega_{n}}{\Omega_{d}} \frac{\mathcal{B}^{2}}{\mathcal{A}^{3}\left(k^{2}\right)} \int d^{d} z Z_{(0 \mid 2)}(z) \square \eta^{2}(z),
$$

where only the terms of second order in the gradient are retained. The first term leads to the contribution

$$
\frac{\hbar k^{d} \alpha_{d}}{2} \int \frac{d^{d} p_{1}}{(2 \pi)^{d}} \frac{\mathcal{B}^{2}}{\mathcal{A}\left(k^{2}\right) \mathcal{A}\left(\left(k n+p_{1}\right)^{2}\right)} \eta_{p_{1}} \eta_{-p_{1}}
$$

after performing $\Sigma_{P}^{\prime}$. Expanding the integrand in powers of $p_{1}^{\mu}$, we find for the contribution (A20)

$$
\begin{aligned}
& \hbar k^{d} \alpha_{d} \frac{1}{2} \int \frac{d \omega_{n}}{\Omega_{d}}\left\{\frac{\mathcal{B}_{0}^{2}}{\mathcal{A}^{2}\left(k^{2}\right)} \int d^{d} z \eta^{2}(z)\right. \\
& \left.-\mathcal{G}_{\rho \sigma} \int d^{d} z \eta(z) \partial^{\rho} \partial^{\sigma} \eta(z)\right\},
\end{aligned}
$$

where

$$
\mathcal{G}_{\rho \sigma}=\frac{\mathcal{B}_{0}^{2} Z_{(1 \mid 1)}(k)}{\mathcal{A}^{3}\left(k^{2}\right)}\left[\frac{4 k^{2}}{\mathcal{A}\left(k^{2}\right)} Z_{(1 \mid 1)}(k) n_{\rho} n_{\sigma}-g_{\rho \sigma}\right]
$$

Adding all the contributions on the RHS of Eq. (A9), we can identify the coefficients of the various composite operators on both of its sides. Removing now the inhomogeneity of the couplings, we find

$$
\begin{aligned}
& k \partial_{k} V^{(2)}\left(\varphi_{0}\right)=-\hbar k^{d} \alpha_{d} \partial_{\varphi_{0}}^{2} \ln \mathcal{A}\left(k^{2}\right), \\
& k \partial_{k} Z_{(1 \mid 1)}(k)=\hbar k^{d} \alpha_{d} \frac{1}{d} \mathcal{G}_{\mu}^{\mu}(0), \\
& k \partial_{k} Z_{(0 \mid 2)}(k)=\hbar k^{d} \alpha_{d}\left[\frac{V^{(4)}(0)}{\mathcal{A}_{0}^{2}}-\frac{2 \mathcal{B}^{2}}{\mathcal{A}_{0}^{3}}\right] Z_{(0 \mid 2)}(k)
\end{aligned}
$$

with $\mathcal{G}_{\mu}^{\mu}(0)=\left.\mathcal{G}_{\mu}^{\mu}\right|_{\varphi_{0}=0}$. Equation (A23) can also be obtained by differentiating both sides of Eq. (A11) with respect to $\varphi_{0}$ two times. Equation (A25) is equivalent with Eq. (A16), whereas Eq. (A24) is an independent equation.

\section{APPENDIX B: SOLUTION OF RG EQUATIONS FOR THE BLOCKED COUPLINGS IN IMA}

We rewrite Eqs. (A11), (A15), (A16), and (A24) in integral form and substitute the bare values $g_{n}(\Lambda)$ for the blocked couplings $g_{n}(k)$ in the integrands. In terms of the integrals $I_{0}$ and $I_{n r s}$ given in Appendix E, we have

$$
V_{k}\left(\varphi_{0}\right)=V_{\Lambda}\left(\varphi_{0}\right)+\hbar \alpha_{d} I_{0}
$$

$Z_{(0 \mid 2)}(k)=Z_{(0 \mid 2)}(\Lambda)-\hbar \alpha_{d}\left(g_{4}(\Lambda) I_{002}-2 I_{023}\right) Z_{(0 \mid 2)}(\Lambda)$,

$Z_{(0 \mid 1)}(k)=Z_{(0 \mid 1)}(\Lambda)-\hbar \alpha_{d} I_{012} Z_{(0 \mid 2)}(\Lambda)$,

$Z_{(1 \mid 1)}(k)=Z_{(1 \mid 1)}(\Lambda)\left[1-\hbar \alpha_{d}\left(\frac{4 Z_{(1 \mid 1)}(\Lambda)}{d} I_{124}-I_{023}\right)\right]$. 
TABLE IV. The mixing matrix elements $s_{m n}^{I M A}(k)$.

\begin{tabular}{lcc}
\hline \hline$m, n$ & Integral form & After integration \\
\hline 0,2 & $\hbar \alpha_{6} \partial_{m^{2} I_{0}(0)=\hbar \alpha_{6} I_{001}}$ & $\hbar \alpha_{6} f_{4}(\Lambda) / 2$ \\
1,2 & $\hbar \alpha_{6} \partial_{m^{2}} I_{0}^{(1)}(0)=-\hbar \alpha_{6} I_{012}$ & $\hbar \alpha_{6} \lambda f_{2}(\Lambda)$ \\
2,2 & $1+\hbar \alpha_{6} \partial_{m^{2}} I_{0}^{(2)}(0)=1+2 \hbar \alpha_{6} I_{023}$ & $1+\hbar \alpha_{6} \lambda^{2} f_{0}(\Lambda)$ \\
3,2 & $\hbar \alpha_{6} \partial_{m^{2}} I_{0}^{(3)}(0)=-6 \hbar \alpha_{6} I_{034}$ & $-\hbar \alpha_{6} \lambda^{3} / m^{2}$ \\
4,2 & $\hbar \alpha_{6} \partial_{m^{2}} I_{0}^{(4)}(0)=24 \hbar \alpha_{6} I_{045}$ & $\hbar \alpha_{6} \lambda^{4} / m^{4}$ \\
1,3 & $\hbar \alpha_{6} \partial_{\lambda} I_{0}^{(1)}(0)=\hbar \alpha_{6} I_{001}$ & $\hbar \alpha_{6} f_{4}(\Lambda) / 2$ \\
2,3 & $\hbar \alpha_{6} \partial_{\lambda} I_{0}^{(2)}(0)=-2 \hbar \alpha_{6} I_{012}$ & $2 \hbar \alpha_{6} \lambda f_{2}(\Lambda)$ \\
3,3 & $1+\hbar \alpha_{6} \partial_{\lambda} I_{0}^{(3)}(0)=1+6 \hbar \alpha_{6} I_{023}$ & $1+3 \hbar \alpha_{6} \lambda^{2} f_{0}(\Lambda)$ \\
4,3 & $\hbar \alpha_{6} \partial_{\lambda} I_{0}^{(4)}(0)=-24 \hbar \alpha_{6} I_{034}$ & $-4 \hbar \alpha_{6} \lambda^{3} / m^{2}$ \\
2,4 & $\hbar \alpha_{6} \partial_{g} I_{0}^{(2)}(0)=\hbar \alpha_{6} I_{001}$ & $\hbar \alpha_{6} f_{4}(\Lambda) / 2$ \\
3,4 & $\hbar \alpha_{6} \partial_{g} I_{0}^{(3)}(0)=-3 \hbar \alpha_{6} I_{012}$ & $3 \hbar \alpha_{6} \lambda f_{2}(\Lambda)$ \\
4,4 & $1+\hbar \alpha_{6} \partial_{g} I_{0}^{(4)}(0)=1+12 \hbar \alpha_{6} I_{023}$ & $1+6 \hbar \alpha_{6} \lambda^{2} f_{0}(\Lambda)$ \\
\hline \hline
\end{tabular}

Let us evaluate the elements $s_{n m}$ of the operator-mixing matrix for the $\phi^{3}$ theory.

\section{From monomials to monomials}

We have from Eq. (B1)

$$
g_{l}(k)=g_{l}(\Lambda)+\hbar \alpha_{d} I_{0}^{(l)}(0),
$$

and, for the mixing coefficients of the monomial operators,

$$
s_{l l^{\prime}}=\frac{\partial g_{l}(k)}{\partial g_{l^{\prime}}(\Lambda)}=\delta_{l l^{\prime}}+\hbar \alpha_{d} \frac{\partial I_{0}^{(l)}(0)}{\partial g_{l^{\prime}}(\Lambda)} .
$$

The partial derivatives on the RHS of Eq. (B5) can be evaluated by taking the partial derivatives of $I_{0}$ (given in Appendix E) with respect to $\varphi_{0}$ at $\varphi_{0}=0$ and then differentiating them with respect to the appropriate $g_{l^{\prime}}(\Lambda)$, before the integration over $\kappa$ is performed. Finally, the initial values of the couplings specifying the $\phi^{3}$ theory should be inserted.

In Table IV we summarize the nontrivial operator mixing coefficients in terms of the integrals given in Appendix $\mathrm{E}$ and their limiting values for $k=0, \Lambda^{2} \gg m^{2}$ after carrying out the loop integral in IMA. The functions $f_{a}, a=0,2,4$ in the last column are defined as

$$
\begin{aligned}
& f_{0}(\Lambda)=\left[\ln \left(\frac{\Lambda^{2}}{m^{2}}+1\right)-\frac{3}{2}\right], \\
& f_{2}(\Lambda)=\left[m^{2} \ln \left(\frac{\Lambda^{2}}{m^{2}}+1\right)-\frac{1}{2} \Lambda^{2}-\frac{1}{2} m^{2}\right], \\
& f_{4}(\Lambda)=\left[m^{4} \ln \left(\frac{\Lambda^{2}}{m^{2}}+1\right)+\frac{1}{2} \Lambda^{4}-\Lambda^{2} m^{2}\right],
\end{aligned}
$$

$$
\begin{aligned}
f_{6}(\Lambda)= & -\frac{1}{3} \Lambda^{6}+\frac{1}{2} \Lambda^{4} m^{2}-\Lambda^{2} m^{4} \\
& +m^{6} \ln \left(\frac{\Lambda^{2}}{m^{2}}+1\right) .
\end{aligned}
$$

The theory is renormalizable and we see that monomial operators $\varphi^{l^{\prime}}$ of dimension not greater than the dimension of the monomial $\varphi^{l}$ are the only ones admixing with the blocked operator $\left\{\varphi^{l}\right\}_{k \rightarrow 0}$ with UV-divergent coefficients. Were the theory nonrenormalizable, e.g., a $\phi^{4}$ term with nonvanishing coupling $g_{4}(\Lambda) \neq 0$ included, then also the admixture of higher-dimensional operators would occur with UV-divergent coefficients.

These results can be compared with the one-loop perturbative result given in $[[6]$ p. $145,(6.2 .11)]$ for the renormalized operator $\frac{1}{2}\left[\phi^{2}\right]_{R}$, replacing $2 /(d-6)$ by $-\ln \left(\Lambda^{2} / m^{2}\right)$ and using $\alpha_{6}=1 /\left(128 \pi^{3}\right.$ ) (in our notations):

$$
\begin{aligned}
\frac{1}{2}\left[\phi^{2}\right]_{R}= & M_{1}(\phi)\left(Z^{-1}\right)_{12}+M_{2}(\phi)\left(Z^{-1}\right)_{22}+D_{(0 \mid 1)}(\phi) \\
& \times\left(Z^{-1}\right)_{(0 \mid 1) 2}+\cdots,
\end{aligned}
$$

where.. stands for higher-order terms and

$$
\begin{aligned}
\left(Z^{-1}\right)_{12} & =-\hbar \frac{\lambda m^{2}}{128 \pi^{3}} \ln \frac{\Lambda^{2}}{m^{2}}, \\
\left(Z^{-1}\right)_{22} & =1-\hbar \frac{\lambda^{2}}{128 \pi^{3}} \ln \frac{\Lambda^{2}}{m^{2}}, \\
\left(Z^{-1}\right)_{(0 \mid 1) 2} & =\hbar \frac{\lambda}{6 \times 128 \pi^{3}} \ln \frac{\Lambda^{2}}{m^{2}} .
\end{aligned}
$$

As far as the mixing of monomials is considered, this illustrates that the operator mixing matrix $s_{n m}$ in the IMA in the limit $k \rightarrow 0$ and $\Lambda^{2} \gg m^{2}$ satisfies the relation $s_{l l^{\prime}}=Z_{l l^{\prime}}$, where $Z_{n m}$ is the one-loop perturbative operator-mixing matrix.

\section{From monomials to derivative operators}

Equation (B5) obtained from Eq. (B1) can also be used to determine the admixture of monomials with the blocked derivative operators in the IMA. Since $I_{0}^{(n)}(0)$ depends only on $z=Z_{(1 \mid 1)}(\Lambda)$ and not on the couplings of the other derivative operators, one obtains $s_{l(r \mid s)}(k)=0$ for $(r \mid s) \neq(1 \mid 1)$, and

$$
s_{l(1 \mid 1)}(k)=\hbar \alpha_{d} \frac{\partial I_{0}^{(n)}(0)}{\partial z} .
$$

The first few nonvanishing operator-mixing coefficients and their limiting values for $k=0, \Lambda^{2} \gg m^{2}$ are collected in Table $\mathrm{V}$. Thus, we can write for the blocked operator (with constant argument): $\left\{D_{(1 \mid 1)}\left(\varphi_{0}\right)\right\}_{k}=\sum_{n=0}^{\infty} s_{n(1 \mid 1)}(k) \varphi_{0}^{n} / n !$. Again, the contributions of the derivative operators to the 
TABLE V. The mixing matrix elements $s_{l(m \mid n)}^{I M A}(k) / \hbar \alpha_{6}$.

\begin{tabular}{lcc}
\hline \hline$l(m \mid n)$ & Integral form & After integration \\
\hline $0(1 \mid 1)$ & $I_{101}$ & $-f_{6}(\Lambda) / 2$ \\
$1(1 \mid 1)$ & $-I_{112}$ & $-\lambda\left[3 f_{4}(\Lambda)-\Lambda^{4}+\Lambda^{2} m^{2}-m^{4}\right] / 6$ \\
$2(1 \mid 1)$ & $2 I_{123}$ & $-\lambda^{2}\left[6 f_{2}(\Lambda)+\Lambda^{2}-2 m^{2}\right]$ \\
$3(1 \mid 1)$ & $-6 I_{134}$ & $-\lambda^{3}\left[3 f_{0}(\Lambda)-1\right]$ \\
$4(1 \mid 1)$ & $24 I_{145}$ & $3 \lambda^{4} / m^{2}$ \\
\hline \hline
\end{tabular}

blocked derivative operator $\left\{D_{(1 \mid 1)}\left(\varphi_{k}\right)\right\}_{k}$ can only be seen if the latter is considered in the general argument $\varphi_{k}(x)$.

\section{From derivative operators to monomials and derivative operators}

Differentiating the solutions (B4), (B2), and (B3) with respect to the various bare couplings, the following additional (nonvanishing) operator-mixing coefficients and their limiting values for $k=0, \Lambda^{2} \gg m^{2}$ are shown in Table VI.

The result obtained for $Z_{(1 \mid 1)}(k=0)$ in the IMA is in agreement with the one-loop perturbative result for $Z_{\phi}$ :

$$
Z_{\phi}=1+\frac{\hbar \lambda^{2}}{12 \cdot 64 \pi^{3}}\left(\ln \frac{\Lambda^{2}}{m^{2}}-\frac{5}{6}\right)
$$

(see [6], p. 58) as expected, since the correspondence $Z_{(1 \mid 1)}(k=0)=Z_{\phi}$ should hold due to

$$
\begin{aligned}
\int d^{d} x \frac{1}{2} Z_{\phi}\left(\partial_{\mu} \phi\right)^{2} & =\int d^{d} x \frac{1}{2} Z_{\phi}\left(2 D_{(1 \mid 1)}-D_{(0 \mid 2)}\right) \\
& =\int d^{d} x Z_{(1 \mid 1)} D_{(1 \mid 1)}
\end{aligned}
$$

Using $Z_{(0 \mid 2)}(\Lambda)=-\frac{1}{2}$ [owing to Eq. (7.4)] and $Z_{(0 \mid 1)}(\Lambda)$ $=0$, a comparison of $s_{(0 \mid 1) 2}$ with the corresponding one-loop perturbative result [see relation (B8) taken from [6]] gives $s_{(0 \mid 1) 2}=3 Z_{(0 \mid 1) 2}$. Since $s_{(0 \mid 1) 2}$ obtained above agrees with our perturbative one-loop result in Appendix D, so that both results contradict to Eq. (B8), there ought to be a misprint in [6].

\section{APPENDIX C: CONNECTION MATRIX}

First, we read off the beta functions from the right-hand sides of Eqs. (A11), (A15), (A16), and (A24). The beta functions for the couplings of the monomials are given by $\beta_{l}(k)=\left.\hbar \alpha_{6} k^{6} \partial_{\varphi_{0}}^{l} \ln \mathcal{A}\left(k^{2}\right)\right|_{\varphi_{0}=0}$, whereas the other beta functions can be read off directly. With the notations $m^{2}(k)=V^{(2)}(0), \quad \lambda(k)=V^{(3)}(0), \quad g(k)=V^{(4)}(0), \quad g_{l}(k)$ $=V^{(l>4)}(0)$, and $G=\left[Z_{(1 \mid 1)}(k) k^{2}+m^{2}(k)\right]^{-1}$, the explicit forms of the beta functions are given in Table VII.

The elements of the connection matrix, shown in Table VIII are obtained by differentiation according to the second equation of Eq. (4.14).

\section{APPENDIX D: PERTURBATIVE ONE-LOOP RESULTS FOR OPERATOR MIXING}

Here we determine the operator-mixing matrix at oneloop order perturbatively for $\lambda \phi^{3}$ theory in dimension $d$ $=6$. Let us decompose the action $S$ into the free part

$$
S_{0}=\int d^{d} x\left[-\frac{1}{2} Z_{R} \phi \square \phi+\frac{1}{2} m_{R}^{2} \phi^{2}\right],
$$

the interaction part

TABLE VI. The mixing matrix elements $s_{\text {index }}^{I M A}(k)$.

\begin{tabular}{lcc}
\hline \hline Index & Integral form & After integration \\
\hline$(1 \mid 1) 2$ & $\hbar \alpha_{6}\left(8 Z_{(1 \mid 1)}^{2} I_{125}-9 Z_{(1 \mid 1)} I_{024}\right) / 3$ & $-\hbar \alpha_{6} \lambda^{2} / 6 m^{2}$ \\
$(1 \mid 1) 3$ & $-\hbar \alpha_{6}\left(4 Z_{(1 \mid 1)}^{2} I_{114}-6 Z_{(1 \mid 1)} I_{013}\right) / 3$ & $\hbar \alpha_{6} \lambda\left[3 f_{0}(\Lambda)+2\right] / 9$ \\
$(1 \mid 1)(1 \mid 1)$ & $1-\hbar \alpha_{6}\left(13 Z_{(1 \mid 1)} I_{124}-3 I_{023}-8 Z_{(1 \mid 1)}^{2} I_{225}\right) / 3$ & $1-\hbar \alpha_{6} \lambda^{2}\left[6 f_{0}(\Lambda)+1\right] / 18$ \\
$(0 \mid 2) 2$ & $-6 \hbar \alpha_{6} Z_{(0 \mid 2)}(\Lambda) I_{024}$ & $-\hbar \alpha_{6} \lambda^{2} Z_{(0 \mid 2)}(\Lambda) / m^{2}$ \\
$(0 \mid 2) 3$ & $4 \hbar \alpha_{6} Z_{(0 \mid 2)}(\Lambda) I_{013}(\Lambda) I_{002}$ & $2 \hbar \alpha_{6} \lambda Z_{(0 \mid 2)}(\Lambda) f_{0}(\Lambda)$ \\
$(0 \mid 2) 4$ & $-\hbar \alpha_{6} Z_{(0 \mid 2)}(\Lambda)$ & $\hbar \alpha_{6} Z_{(0 \mid 2)}(\Lambda) f_{2}(\Lambda)$ \\
$(0 \mid 2)(0 \mid 2)$ & $1+2 \hbar \alpha_{6} I_{023}$ & $1+\hbar \alpha_{6} \lambda^{2} f_{0}(\Lambda)$ \\
$(0 \mid 2)(1 \mid 1)$ & $-6 \hbar \alpha_{6} Z_{(0 \mid 2)}(\Lambda) I_{124}$ & $-3 \hbar \alpha_{6} \lambda^{2} Z_{(0 \mid 2)}(\Lambda)\left[3 f_{0}(\Lambda)+2\right] / 3$ \\
$(0 \mid 1) 2$ & $2 \hbar \alpha_{6} Z_{(0 \mid 2)}(\Lambda) I_{013}$ & $\hbar \alpha_{6} \lambda Z_{(0 \mid 2)}(\Lambda) f_{0}(\Lambda)$ \\
$(0 \mid 1) 3$ & $-\hbar \alpha_{6} Z_{(0 \mid 2)}(\Lambda) I_{002}$ & $\hbar \alpha_{6} Z_{(0 \mid 2)}(\Lambda) f_{2}(\Lambda)$ \\
$(0 \mid 1)(0 \mid 1)$ & 1 & 1 \\
$(0 \mid 1)(0 \mid 2)$ & $-\hbar \alpha_{6} I_{012}$ & $\hbar \alpha_{6} \lambda f_{2}(\Lambda)$ \\
$(0 \mid 1)(1 \mid 1)$ & $2 \hbar \alpha_{6} Z_{(0 \mid 2)}(\Lambda) I_{113}$ & $-\hbar \alpha_{6} \lambda Z_{(0 \mid 2)}(\Lambda)\left[6 f_{2}(\Lambda)+\Lambda^{2}-2 m^{2}\right] / 2$ \\
\hline \hline
\end{tabular}


TABLE VII. The beta functions $\beta_{\text {index }}(k) / \hbar \alpha_{6} k^{6}$.

\begin{tabular}{lc}
\hline \hline Index & Beta function \\
\hline 0 & $-\ln G$ \\
1 & $G \lambda$ \\
2 & $G\left(g-G \lambda^{2}\right)$ \\
$(0 \mid 1)$ & $G^{2} \lambda Z_{(0 \mid 2)}$ \\
3 & $G\left(g_{5}-3 g G \lambda+2 G^{2} \lambda^{3}\right)$ \\
$(0 \mid 2)$ & $G^{2} Z_{(0 \mid 2)}(g-2 G \lambda)$ \\
$(1 \mid 1)$ & $G^{3} \lambda^{2} Z_{(1 \mid 1)}\left(2 k^{2} G Z_{(1 \mid 1)}-3\right) / 3$ \\
4 & $G\left(g_{6}-4 g_{5} G \lambda-3 g^{2} G+12 g G^{2} \lambda^{2}-6 G^{3} \lambda^{4}\right)$ \\
\hline \hline
\end{tabular}

$$
\begin{aligned}
S_{I}= & \int d^{d} x\left[-\frac{1}{2} g_{(1 \mid 1) R}(x) \phi \square \phi+\frac{1}{2} g_{2 R}(x) \phi^{2}\right. \\
& \left.+\sum_{n \neq(1 \mid 1), 2} G_{n R}(x) O_{n}[\phi(x)]\right],
\end{aligned}
$$

and the counterterms

TABLE VIII. The nonvanishing connection matrix elements

\begin{tabular}{|c|c|}
\hline Index & Connection matrix \\
\hline 02 & $G$ \\
\hline $0(1 \mid 1)$ & $k^{2} G$ \\
\hline 12 & $-G^{2} \lambda$ \\
\hline 13 & $G$ \\
\hline $1(1 \mid 1)$ & $-k^{2} G^{2} \lambda$ \\
\hline 22 & $G^{2}\left(-g+2 G \lambda^{2}\right)$ \\
\hline 23 & $-2 G^{2} \lambda$ \\
\hline $2(1 \mid 1)$ & $k^{2} G^{2}\left(-g+2 G \lambda^{2}\right)$ \\
\hline 24 & $G$ \\
\hline$(0 \mid 1) 2$ & $-2 G^{3} \lambda Z_{(0 \mid 2)}$ \\
\hline$(0 \mid 1) 3$ & $G^{2} Z_{(0 \mid 2)}$ \\
\hline$(0 \mid 1)$ & $G^{2} \lambda$ \\
\hline$(0 \mid 1)(1 \mid 1)$ & $-2 k^{2} G^{3} \lambda Z_{(0 \mid 2)}$ \\
\hline 32 & $-G^{2}\left(g_{5}-6 g G \lambda+6 G^{2} \lambda^{3}\right)$ \\
\hline 33 & $-G^{2}\left(3 g-6 G \lambda^{2}\right)$ \\
\hline $3(1 \mid 1)$ & $-k^{2} G^{2}\left(g_{5}-6 g G \lambda+6 G^{2} \lambda^{3}\right)$ \\
\hline 34 & $-3 G^{2} \lambda$ \\
\hline$(0 \mid 2) 2$ & $2 G^{3} Z_{(0 \mid 2)}\left(g+3 G \lambda^{2}\right)$ \\
\hline$(0 \mid 2) 3$ & $-4 G^{3} \lambda Z_{(0 \mid 2)}$ \\
\hline$(0 \mid 2)(0 \mid 2)$ & $G^{2}\left(g-2 G \lambda^{2}\right)$ \\
\hline$(0 \mid 2)(1 \mid 1)$ & $-2 k^{2} G^{3} Z_{(0 \mid 2)}\left(g-3 G \lambda^{2}\right)$ \\
\hline$(0 \mid 2) 4$ & $G^{2} Z_{(0 \mid 2)}$ \\
\hline$(1 \mid 1) 2$ & $G^{4} \lambda^{2} Z_{(1 \mid 1)}\left(9-8 k^{2} G Z_{(1 \mid 1)}\right) / 3$ \\
\hline$(1 \mid 1) 3$ & $G^{3} \lambda Z_{(1 \mid 1)}\left(4 k^{2} G Z_{(1 \mid 1)}-6\right) / 3$ \\
\hline$(1 \mid 1)(1 \mid 1)$ & $G^{3} \lambda^{2}\left(12 k^{2} G Z_{(1 \mid 1)}-3-8 k^{4} G^{2} Z_{(1 \mid 1)}^{2}\right) / 3$ \\
\hline 42 & $G^{2}\left(-g_{6}+8 g_{5} G \lambda+6 g^{2} G-36 g G^{2} \lambda^{2}+24 G^{3} \lambda^{4}\right)$ \\
\hline 43 & $-4 G^{2}\left(g_{5}-6 g G \lambda+6 G^{2} \lambda^{3}\right)$ \\
\hline $4(1 \mid 1)$ & $-k^{2} G^{2}\left(g_{6}-8 \lambda g_{5} G \lambda-6 g^{2} G+36 g G^{2} \lambda^{2}-24 G^{3} \lambda^{4}\right)$ \\
\hline 44 & $-6 G^{2}\left(g-2 G \lambda^{2}\right)$ \\
\hline
\end{tabular}
$k \gamma_{\text {index }}(k) / \hbar \alpha_{6} k^{6}$.
TABLE IX. The counterterms expressed in terms of the integrals with $\lambda=g_{3 R}$.

\begin{tabular}{lc}
\hline \hline Index & $c_{\text {index }} / \hbar \alpha_{d}$ \\
\hline 02 & $G$ \\
1 & $-I_{011}$ \\
2 & $I_{022}-g_{4 R} I_{001}$ \\
$(0 \mid 1)$ & $Z_{(0 \mid 2) R} I_{012}$ \\
3 & $3 g_{4 R} I_{012}-2 I_{033}$ \\
$(0 \mid 2)$ & $\left(g_{4 R} I_{002}-2 I_{023}\right) Z_{(0 \mid 2) R}$ \\
$(1 \mid 1)$ & $\frac{4 Z_{R}^{2}}{d} I_{124}-Z_{R} I_{023}$ \\
4 & $3 g_{4 R}^{2} I_{002}-12 g_{4 R} I_{023}+6 I_{044}$ \\
\hline \hline
\end{tabular}

$$
S_{c . t .}=\int d^{d} x \sum_{n} c_{n}\left(g_{m R}\right) O_{n}[\phi(x)] .
$$

Here the same base operators are taken into account as in the blocked action (7.3). The one-loop effective action is given by

$$
\begin{aligned}
\Gamma_{1}= & \frac{\hbar}{2} \operatorname{Tr} \ln \left[1_{p q}+G_{R}\left(p^{2}\right)\left(S_{I}^{(2)}\right)_{-p} q\right] \\
= & -\frac{\hbar}{2} \sum_{v=1}^{\infty} \frac{(-1)^{v}}{v} \int_{p_{1}} \cdots \int_{p_{v}} G_{R}\left(p_{1}^{2}\right) \\
& \times\left(S_{I}^{(2)}\right)_{-p_{1} p_{2}} \cdots G_{R}\left(p_{v}^{2}\right)\left(S_{I}^{(2)}\right)_{-p_{v} p_{1}}
\end{aligned}
$$

with $G_{R}\left(p^{2}\right)=\left(Z_{R} p^{2}+m_{R}^{2}\right)^{-1}$ and the second functional derivative of the interaction piece of the action:

$$
\begin{aligned}
\left(S_{I}^{(2)}\right)_{p q}= & \int d x e^{i(p+q) x}\left[g_{2 R}(x)+g_{(1 \mid 1) R}(x) \frac{1}{2}\left(p^{2}+q^{2}\right)\right. \\
& +\sum_{l=3}^{\infty}\left[g_{l R}+g_{l R}(x)\right] \frac{1}{(l-2) !} \phi^{l-2}(x) \\
& \left.+\left[Z_{(0 \mid 2) R}+g_{(0 \mid 2) R}(x)\right](p+q)^{2}\right]
\end{aligned}
$$

In the second order of the derivative expansion, the counterterms are defined through the relations

$$
\begin{aligned}
-\left.\frac{\delta \Gamma_{1}}{\delta \varphi(p)}\right|_{\phi, g \tilde{n} R}=0 & {\left[c_{1}+c_{(0 \mid 1)} p^{2}+o\left(p^{4}\right)\right] } \\
& \times(2 \pi)^{d} \delta(p) \\
-\left.\frac{\delta^{2} \Gamma_{1}}{\delta \varphi\left(p_{1}\right) \delta \varphi\left(p_{2}\right)}\right|_{\phi, g \tilde{n} R}= & \left(c_{2}+c_{(1 \mid 1)} p_{1}^{2}+o\left(p_{1}^{4}\right)\right)(2 \pi)^{d} \\
& \times \delta\left(p_{1}+p_{2}\right),
\end{aligned}
$$


TABLE X. The operator-mixing matrix $\left(Z^{-1}\right)_{\text {index }}$.

\begin{tabular}{|c|c|}
\hline Index & Mixing matrix \\
\hline 10,11 & 1 \\
\hline 12 & $\hbar \alpha_{d} I_{012}$ \\
\hline $1(0 \mid 1)$ & 0 \\
\hline 13 & $-\hbar \alpha_{d} I_{001}$ \\
\hline $1(0 \mid 2)$ & 0 \\
\hline $1(1 \mid 1)$ & $\hbar \alpha_{d} I_{112}$ \\
\hline 14 & 0 \\
\hline 20,21 & 0 \\
\hline 22 & $1+\hbar \alpha_{d}\left(g_{4 R} I_{002}-2 I_{023}\right)$ \\
\hline $2(0 \mid 1)$ & 0 \\
\hline 23 & $2 \hbar \alpha_{d} I_{012}$ \\
\hline $2(0 \mid 2)$ & 0 \\
\hline $2(1 \mid 1)$ & $\hbar \alpha_{d}\left(g_{4 R} I_{102}-2 I_{123}\right)$ \\
\hline 24 & $-\hbar \alpha_{d} I_{001}$ \\
\hline$(0 \mid 1) 0,(0 \mid 1) 1$ & 0 \\
\hline$(0 \mid 1) 2$ & $-2 \hbar \alpha_{d} I_{013} Z_{(0 \mid 2) R}$ \\
\hline$(0 \mid 1)(0 \mid 1)$ & 1 \\
\hline$(0 \mid 1) 3$ & $\hbar \alpha_{d} I_{002} Z_{(0 \mid 2) R}$ \\
\hline$(0 \mid 1)(0 \mid 2)$ & $\hbar \alpha_{d} I_{012}$ \\
\hline$(0 \mid 1)(1 \mid 1)$ & $-2 \hbar \alpha_{d} I_{113} Z_{(0 \mid 2) R}$ \\
\hline$(0 \mid 1) 4$ & 0 \\
\hline 30,31 & 0 \\
\hline 32 & $6 \hbar \alpha_{d}\left(I_{034}-g_{4 R} I_{013}\right)$ \\
\hline $3(0 \mid 1)$ & 0 \\
\hline 33 & $1+3 \hbar \alpha_{d}\left(g_{4 R} I_{002}-2 I_{023}\right)$ \\
\hline $3(0 \mid 2)$ & 0 \\
\hline $3(1 \mid 1)$ & $6 \hbar \alpha_{d}\left(I_{134}-g_{4 R} I_{113}\right)$ \\
\hline 34 & $3 \hbar \alpha_{d} I_{012}$ \\
\hline$(0 \mid 2) 0,(0 \mid 2) 1$ & 0 \\
\hline$(0 \mid 2) 2$ & $\hbar \alpha_{d}\left(6 I_{024}-2 g_{4 R} I_{003}\right) Z_{(0 \mid 2) R}$ \\
\hline$(0 \mid 2)(0 \mid 1)$ & 0 \\
\hline$(0 \mid 2) 3$ & $-4 \hbar \alpha_{d} I_{013} Z_{(0 \mid 2) R}$ \\
\hline$(0 \mid 2)(0 \mid 2)$ & $1+\hbar \alpha_{d}\left(g_{4 R} I_{002}-2 I_{023}\right)$ \\
\hline$(0 \mid 2)(1 \mid 1)$ & $\hbar \alpha_{d}\left(6 I_{124}-2 g_{4 R} I_{103}\right) Z_{(0 \mid 2) R}$ \\
\hline$(0 \mid 2) 4$ & $\hbar \alpha_{d} I_{002} Z_{(0 \mid 2) R}$ \\
\hline$(1 \mid 1) 0,(1 \mid 1) 1$ & 0 \\
\hline$(1 \mid 1) 2$ & $\hbar \alpha_{d}\left(3 d Z_{R} I_{024}-16 Z_{R}^{2} I_{125}\right) / d$ \\
\hline$(1 \mid 1)(0 \mid 1)$ & 0 \\
\hline$(1 \mid 1) 3$ & $\hbar \alpha_{d}\left(8 Z_{R}^{2} I_{114}-2 d Z_{R} I_{013}\right) / d$ \\
\hline$(1 \mid 1)(0 \mid 2)$ & 0 \\
\hline$(1 \mid 1)(1 \mid 1)$ & $1+\hbar \alpha_{d}\left[-16 Z_{R}^{2} I_{225}+(8+3 d) Z_{R} I_{124}-d I_{023}\right] / d$ \\
\hline$(1 \mid 1) 4$ & 0 \\
\hline 40,41 & 0 \\
\hline 42 & $6 \hbar \alpha_{d}\left(-g_{4 R}^{2} I_{003}+6 g_{4 R} I_{024}-4 I_{045}\right)$ \\
\hline $4(0 \mid 1)$ & 0 \\
\hline 43 & $24 \hbar \alpha_{d}\left(I_{034}-g_{4 R} I_{013}\right)$ \\
\hline $4(0 \mid 2)$ & 0 \\
\hline $4(1 \mid 1)$ & $6 \hbar \alpha_{d}\left(-g_{4 R}^{2} I_{103}+6 g_{4 R} I_{124}-4 I_{145}\right)$ \\
\hline 44 & $1+6 \hbar \alpha_{d}\left(g_{4 R} I_{002}-2 I_{023}\right)$ \\
\hline
\end{tabular}

$$
\begin{aligned}
& -\left.\frac{\delta^{s} \Gamma_{1}}{\delta \varphi\left(p_{1}\right) \cdots \delta \varphi\left(p_{s}\right)}\right|_{\phi, g_{\tilde{n} R}=0, p_{i}^{2}=0} \\
& =c_{s}(2 \pi)^{d} \delta\left(p_{1}+\ldots+p_{s}\right), \quad(s \geqslant 3), \\
& -\left.Z_{(0 \mid 2) R} \frac{\delta^{3} \Gamma_{1}}{\delta \varphi\left(p_{1}\right) \delta \varphi\left(p_{2}\right) \delta g_{(0 \mid 2) R}(x)}\right|_{\phi, g_{\tilde{n} R}=0} \\
& =c_{(0 \mid 2)}\left(p_{1}+p_{2}\right)^{2} e^{i\left(p_{1}+p_{2}\right) x}
\end{aligned}
$$

A straightforward evaluation results in the expressions for the counterterms given in Table IX.

For the elements of the operator mixing matrix (6.11) we obtain, with $Z_{R} \equiv Z_{(1 \mid 1) R}=1, Z_{(0 \mid 2) R}=-\frac{1}{2}, g_{n R}=0$ for $n$ $\neq 2,(1 \mid 1),(0 \mid 2), 3$, the expressions shown in Table $X$.

\section{APPENDIX E: PARTICULAR INTEGRALS}

In order to obtain the explicit forms of the solutions of Eqs. (B1), (B4), (B3), and (B2) the following integrals are needed:

$$
\begin{gathered}
I_{0}=\int_{k}^{\Lambda} d p p^{d-1} \ln \left[z p^{2}+V_{\Lambda}^{(2)}\left(\varphi_{0}\right)\right] \\
I_{n r s}=\lambda_{\Lambda}^{r} \int_{k}^{\Lambda} d p p^{d-1} p^{2 n} G_{\Lambda}^{s}=\frac{\lambda_{\Lambda}^{r}}{2 z^{s}} \sum_{i=0}^{j+n-1}\left(\begin{array}{c}
j+n-1 \\
i
\end{array}\right) \\
\times\left(-\frac{m_{\Lambda}^{2}}{z}\right)^{j+n-1-i} \int_{k^{2}}^{\Lambda^{2}} d u\left(u+\frac{m_{\Lambda}^{2}}{z}\right)^{i-s}
\end{gathered}
$$

for even dimensions $d=2 j$, where $G_{\Lambda}=\left[z p^{2}+m_{\Lambda}^{2}\right]^{-1}$. For dimensions $d=6$ we obtain

$$
\begin{aligned}
I_{0}= & \frac{1}{6}\left[u^{3} \ln \left[z u+V_{\Lambda}^{(2)}\left(\varphi_{0}\right)\right]-\frac{1}{3}(u+c)^{3}+\frac{3}{2} c(u+c)^{2}\right. \\
& \left.-3 c^{2} u+c^{3} \ln (u+c)\right]_{u=k^{2}}^{u=\Lambda^{2}}
\end{aligned}
$$

with $c=V_{\Lambda}^{(2)}\left(\varphi_{0}\right) / z$. The evaluation of the integrals $I_{n r s}$ is straightforward. 
[1] K.G. Wilson and J. Kogut, Phys. Rep. 12, 75 (1974).

[2] F.J. Wegner and A. Houghton, Phys. Rev. A 8, 401 (1973).

[3] S. Weinberg, The Quantum Theory of Fields (Cambridge University Press, Cambridge, England, 1996), Vol. II.

[4] S. Pokorski, Gauge Field Theories (Cambridge University Press, Cambridge, England, 1987).

[5] J. Zinn-Justin, Quantum Field Theory and Critical Phenomena (Clarendon Press, Oxford, 1989).

[6] J. Collins, Renormalization (Cambridge University Press, Cambridge, England, 1984).

[7] J. Polchinski, Nucl. Phys. B231, 269 (1984).

[8] J. Hughes and J. Liu, Nucl. Phys. B307, 183 (1988); J. Hughes, ibid. B312, 125 (1989).

[9] G.M. Shore, Nucl. Phys. B362, 85 (1991).

[10] G. Keller and C. Kopper, Commun. Math. Phys. 148, 445 (1992); 153, 254 (1993).

[11] C. Becchi, lectures given at the Parma Theoretical Physics Seminar, 1991, hep-th/9607188.

[12] B.P. Dolan, Int. J. Mod. Phys. A 10, 2439 (1995).

[13] B.P. Dolan, Int. J. Mod. Phys. A 10, 2703 (1995).
[14] B.P. Dolan, Int. J. Mod. Phys. A 12, 2413 (1997).

[15] D.C. Brody and A. Ritz, Nucl. Phys. B522, 588 (1998).

[16] B.P. Dolan and A. Lewis, Phys. Lett. B 460, 302 (1999).

[17] J. Alexandre, V. Branchina, and J. Polonyi, Phys. Rev. D 58, 016002 (1998).

[18] C. Wetterich, Phys. Lett. B 301, 90 (1993); M. Reuter and C. Wetterich, Nucl. Phys. B391, 147 (1993); T.R. Morris, Int. J. Mod. Phys. A 9, 2411 (1994); U. Ellwanger, Phys. Lett. B 335, 364 (1994); N. Tetradis and D.F. Litim, Nucl. Phys. B464, 492 (1996); J. Adams, J. Berges, S. Bornholdt, F. Freire, N. Tetradis, and C. Wetterich, Mod. Phys. Lett. A 10, 2367 (1995).

[19] C.M. Fraser, Z. Phys. C 28, 101 (1985).

[20] T.R. Morris and J.F. Tighe, J. High Energy Phys. 08, 007 (1999); T.R. Morris and M.D. Turner, Nucl. Phys. B509, 637 (1998); T.R. Morris, ibid. B495, 477 (1997); Int. J. Mod. Phys. B 12, 1343 (1998); Nucl. Phys. B (Proc. Suppl.) 42, 811 (1995).

[21] J. Alexandre and J. Polonyi, "'Renormalization Group for the Internal Space,’ hep-th/9902144. 